\title{
Two-component galaxy models with a central BH - II. The ellipsoidal case
}

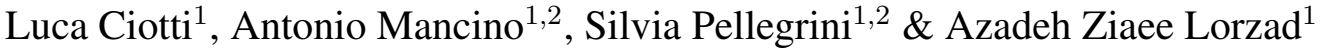 \\ ${ }^{1}$ Department of Physics and Astronomy, University of Bologna, via Gobetti 93/3, 40129 Bologna, Italy \\ ${ }^{2}$ Istituto Nazionale di Astrofisica (INAF), Osservatorio di Astrofisica e Scienza dello Spazio di Bologna (OAS), Via Gobetti 93/3, Bologna 40129, Italy
}

Accepted 2020 October 19. Received 2020 September 29; in original form 2020 July 31

\begin{abstract}
Recently, two-component spherical galaxy models have been presented, where the stellar profile is described by a Jaffe law, and the total density by another Jaffe law, or by an $r^{-3}$ law at large radii. We extend these two families to their ellipsoidal axisymmetric counterparts: the JJe and J3e models. The total and stellar density distributions can have different flattenings and scale lengths, and the dark matter halo is defined by difference. First, the analytical conditions required to have a nowhere negative dark matter halo density are derived. The Jeans equations for the stellar component are then solved analytically, in the limit of small flattenings, also in presence of a central BH. The azimuthal velocity dispersion anisotropy is described by the Satoh $k$-decomposition. Finally, we present the analytical formulae for velocity fields near the center and at large radii, together with the various terms entering the Virial Theorem. The JJe and J3e models can be useful in a number of theoretical applications, e.g. to explore the role of the various parameters (flattening, relative scale lengths, mass ratios, rotational support) in determining the behavior of the stellar kinematical fields before performing more time-expensive integrations with specific galaxy models, to test codes of stellar dynamics, and in numerical simulations of gas flows in galaxies.
\end{abstract}

Key words: methods: analytical - galaxies: kinematics and dynamics - galaxies: structure - galaxies: elliptical and lenticular, $\mathrm{cD}$

\section{INTRODUCTION}

Axially symmetrical models of galaxies are useful tools in Stellar Dynamics (see e.g. BT08), and are often adopted to investigate the presence of dark matter halos (hereafter DM), or central black holes (hereafter BHs), or to study the orbital structure of these systems.

In this paper we extend to the ellipsoidal axisymmetric case two families of two-component (stars plus DM) spherical galaxy models that have been recently presented. In the first family of spherical models (JJ models; Ciotti \& Ziaee Lorzad 2018, hereafter CZ18) the stellar density profile is described by a Jaffe (1983) law, while the total is another spherical Jaffe model of larger total mass and different scale length. In the second family (J3 models; Ciotti, Mancino \& Pellegrini 2019, hereafter CMP19) the stellar density follows again a Jaffe model, while the total is a spherical density profile with a logarithmic slope equal to -3 at large radii. Therefore, the total mass is finite in the $\mathrm{JJ}$ models, and infinite in the $\mathrm{J} 3$ ones. In addition, as supermassive BHs with a mass of the order of $M_{\mathrm{BH}} \simeq 10^{-3} M_{*}$ are generally found at the center of stellar spheroids of total mass $M_{*}$ (see e.g. Magorrian et al. 1988; Kormendy \& Ho 2013), in both models a $\mathrm{BH}$ is added at the center of the galaxy.

In CZ18 it was shown that it is always possible to choose a total mass so that the DM halo resulting from the difference between the total and the stellar density distributions reproduces remarkably well the Navarro-Frenk-White profile (Navarro, Frenk \& White 1997, hereafter NFW) in the inner region. This interesting possibility was further improved in CMP19, where it was proved that the DM halo in the so-called minimum halo model can be tuned to reproduce very well the NFW profile over the whole radial range. Summarizing, JJ and $\mathrm{J} 3$ models present several interesting features, such as analytical simplicity, flexibility in the choice of the structural parameters, realistic stellar and DM density profiles, and fully analytical solutions for the Jeans equations even in presence of a central BH. It is then natural to explore the possibility of a generalization of these spherical models to ellipsoidal (axisymmetrical) shapes: we call the new models JJe and J3e, respectively. Some additional considerations are in order. The first concerns the positivity of the DM halo obtained as the difference of two ellipsoidal distributions with different flattenings and scale lengths. Quite surprisingly, we find that the problem can be solved analytically, and the constraints on the model parameters in order to have a positive DM can be expressed via extremely simple algebraic relations. As a consequence, the positivity problem in JJe and J3e models does not require numerical investigations. The second consideration is about the solution of the Jeans equations. As shown in CZ18 and CMP19, in the spherical case they can be solved analytically, but of course in ellipsoidal models this cannot be expected to be true. In general, to solve them for JJe and $\mathrm{J} 3 \mathrm{e}$ models requires the use of numerical codes (see Caravita et al. 2020 , in preparation). However, in the limit of small flattening, density and potential of ellipsoidal distributions can be expanded at the desired order in the flattening by using the homoeoidal expansion method. Here we show that in this limit the Jeans equations for the homoeoidally expanded JJe and J3e models can be solved analytically (although, not unexpectedly, the formulae are more complicated than that in the sperical case). This possibility to study and plot the resulting kinematical fields, and also to have the quantities entering the Virial Theorem expressed in analytical form for realistic 
ellipsoidal two-component models, without the need for resorting to numerical time-expensive integrations, is a very useful property of $\mathrm{JJe}$ and $\mathrm{J} 3 \mathrm{e}$ models.

The models here introduced, in addition to the standard applications, can be useful in the building of hydrostatic, barotropic and baroclinic models for hot rotating atmospheres in galaxies (see e.g. Barnabè et. al 2006). Moreover, they can be adopted in hydrodynamical simulations of gas flows in galaxies, where the stellar velocity fields are major ingredients in the description of the energy and momentum source terms due to the evolving stellar populations (see e.g. Posacki et al. 2013; Negri et. al 2014).

The paper is organized as follows. In Section 2 the main structural properties of the models are presented, and in Section 3 we set up and discuss the associated Jeans equations. In Section 4 the solution of the Jeans equations is presented, while in Section 5 the asymptotic behaviours of the projected velocity profile at small and large radii are discussed. In Section 6 the Virial Theorem is presented, and the global energetics is explicitly calculated. The main results are finally summarized in Section 7, while the Appendices contain technical details and formulae.

\section{THE MODELS}

The ellipsoidal JJ models (hereafter, JJe models) and the ellipsoidal J3 models (hereafter, J3e models) are the natural generalization of the spherically symmetric JJ and J3 models introduced and fully discussed in CZ18 and CMP19, respectively. It is however useful to recall how these spherical models are defined. The stellar density profile

$$
\rho_{*}(r)=\frac{\rho_{\mathrm{n}}}{s^{2}(1+s)^{2}}
$$

is the same for the two families, where

$$
\rho_{\mathrm{n}} \equiv \frac{M_{*}}{4 \pi r_{*}^{3}}, \quad s \equiv \frac{r}{r_{*}},
$$

and $M_{*}$ and $r_{*}$ are the total stellar mass and the stellar scale length, respectively. The normalization potential and the $\mathrm{BH}$-to-stellar mass ratio are defined as

$$
\Psi_{\mathrm{n}} \equiv \frac{G M_{*}}{r_{*}}, \quad \mu \equiv \frac{M_{\mathrm{BH}}}{M_{*}} .
$$

Following CZ18 and CMP19 the total density profiles can be written as

$$
\rho_{\mathrm{g}}(r)=\rho_{\mathrm{n}} \times \begin{cases}\frac{\mathcal{R} \xi}{s^{2}(\xi+s)^{2}}, & (\mathrm{JJ}), \\ \frac{\mathcal{R}}{s^{2}(\xi+s)}, & (\mathrm{J} 3),\end{cases}
$$

where $\xi \equiv r_{\mathrm{g}} / r_{*}$ is the galaxy scale length $r_{\mathrm{g}}$ in units of $r_{*}$, and $\mathcal{R}$ is a measure of the total-to-stellar density: in particular, for JJ models $\mathcal{R}=M_{\mathrm{g}} / M_{*}$, while for $\mathrm{J} 3$ models $\mathcal{R} / \xi$ can be interpreted as the ratio of the total over the stellar density at the center. Of course, this latter interpretation holds also for the JJ models. The cumulative galactic mass inside a sphere of radius $r$ reads

$$
M_{\mathrm{g}}(r)=M_{*} \times\left\{\begin{array}{l}
\frac{\mathcal{R} s}{\xi+s}, \\
\mathcal{R} \ln \frac{\xi+s}{\xi},
\end{array}\right.
$$

Note that, while $M_{\mathrm{g}}(r)$ tends to a finite value in the case of JJ models, for J3 models it diverges logarithmically. Finally, the (relative) galaxy potential is given by

$$
\Psi_{\mathrm{g}}(r)=\Psi_{\mathrm{n}} \times\left\{\begin{array}{l}
\frac{\mathcal{R}}{\xi} \ln \frac{\xi+s}{s}, \\
\frac{\mathcal{R}}{\xi} \ln \frac{\xi+s}{s}+\frac{\mathcal{R}}{s} \ln \frac{\xi+s}{\xi},
\end{array}\right.
$$

In the present approach, the DM halo density distribution is obtained as $\rho_{\mathrm{DM}}(r)=\rho_{\mathrm{g}}(r)-\rho_{*}(r)$.

We can now introduce the new ellipsoidal models discussed in this paper. The stellar component of JJe and J3e models is given by the mass-conserving ellipsoidal generalization of equation (1),

$$
\rho_{*}(R, z)=\frac{\rho_{\mathrm{n}}}{q_{*} m_{*}^{2}\left(1+m_{*}\right)^{2}}, \quad m_{*}^{2}=\tilde{R}^{2}+\frac{\tilde{z}^{2}}{q_{*}^{2}},
$$

where $q_{*}=1-\eta_{*}$ measures the flattening of the density distribution, and $\tilde{R} \equiv R / r_{*}$ and $\tilde{z} \equiv z / r_{*}$ are the dimensionless cylindrical coordinates. From volume integration of equation (7) the independence of the total mass $M_{*}$ on $q_{*}$ can be immediately verified. In analogy with equation (4), for the total galaxy density profile we assume

$$
\rho_{\mathrm{g}}(R, z)=\rho_{\mathrm{n}} \times \begin{cases}\frac{\mathcal{R} \xi}{q_{\mathrm{g}} m_{\mathrm{g}}^{2}\left(\xi+m_{\mathrm{g}}\right)^{2}}, & (\mathrm{JJe}), \\ \frac{\mathcal{R}}{q_{\mathrm{g}} m_{\mathrm{g}}^{2}\left(\xi+m_{\mathrm{g}}\right)}, & (\mathrm{J} 3 \mathrm{e}),\end{cases}
$$

where $m_{\mathrm{g}}^{2}=\tilde{R}^{2}+\tilde{z}^{2} / q_{\mathrm{g}}^{2}$, and $q_{\mathrm{g}}=1-\eta_{\mathrm{g}}$ is the axial ratio of the total density profile. Of course, when $q_{*}=q_{\mathrm{g}}=1$, JJe and $\mathrm{J} 3 \mathrm{e}$ models reduce respectively to $\mathrm{JJ}$ and $\mathrm{J} 3$ models. As expected, the total mass $M_{\mathrm{g}}(R, z)$ converges to a finite $M_{\mathrm{g}}$ in JJe models, and diverges in $\mathrm{J} 3 \mathrm{e}$ case. It is important to note that for $q_{\mathrm{g}} \neq q_{*}$, i.e. in the case of different flattenings for the total and stellar densities, $\rho_{\mathrm{DM}}$ is $n o t$ stratified on ellipsoidal surfaces.

The projected stellar density associated with equation (7) can be easily obtained from ellipsoidal projection. In particular, when the line-of-sight is inclined by an angle $i$ measured from the $z$-axis (for example, with rotation around the $y$-axis), the projected density is

$$
\Sigma_{*}(\ell)=\frac{M_{*}}{r_{*}^{2} q_{*}(i)} f(\ell), \quad q_{*}(i) \equiv \sqrt{\cos ^{2} i+q_{*}^{2} \sin ^{2} i},
$$

(see e.g. Riciputi et al. 2005), where the function $f(\ell)$ is given in equation 6 of CZ18, and the isodensity $(\ell)$ in the projection plane $(X, Y)$ is

$$
\ell^{2}=\frac{X^{2}}{r_{*}^{2} q_{*}^{2}(i)}+\frac{Y^{2}}{r_{*}^{2}},
$$

so that $q_{*}(i)$ is the "isophotal" flattening (see Section 5). The projected stellar mass contained inside the ellipse defined by $\ell$ is

$$
M_{\mathrm{p} *}(\ell)=M_{*} \times g(\ell),
$$

where $g(\ell)$ is again the same function as in the spherical case (see CZ18, equation 9). In particular, the effective ellipse corresponds to $g\left(\ell_{e}\right)=1 / 2$, i.e. $\ell_{e} \simeq 0.7447$, with a circularized radius (i.e., the radius of the circle in the projection plane with the same area of the effective ellipse) given by $\left\langle R_{\mathrm{e}}\right\rangle \simeq 0.7447 r_{*} \sqrt{q_{*}(i)}$.

\subsection{The dark matter halo: positivity}

As the DM component is given by a difference of two density distributions, a preliminary study of the positivity of its density $\rho_{\mathrm{DM}}$ as 

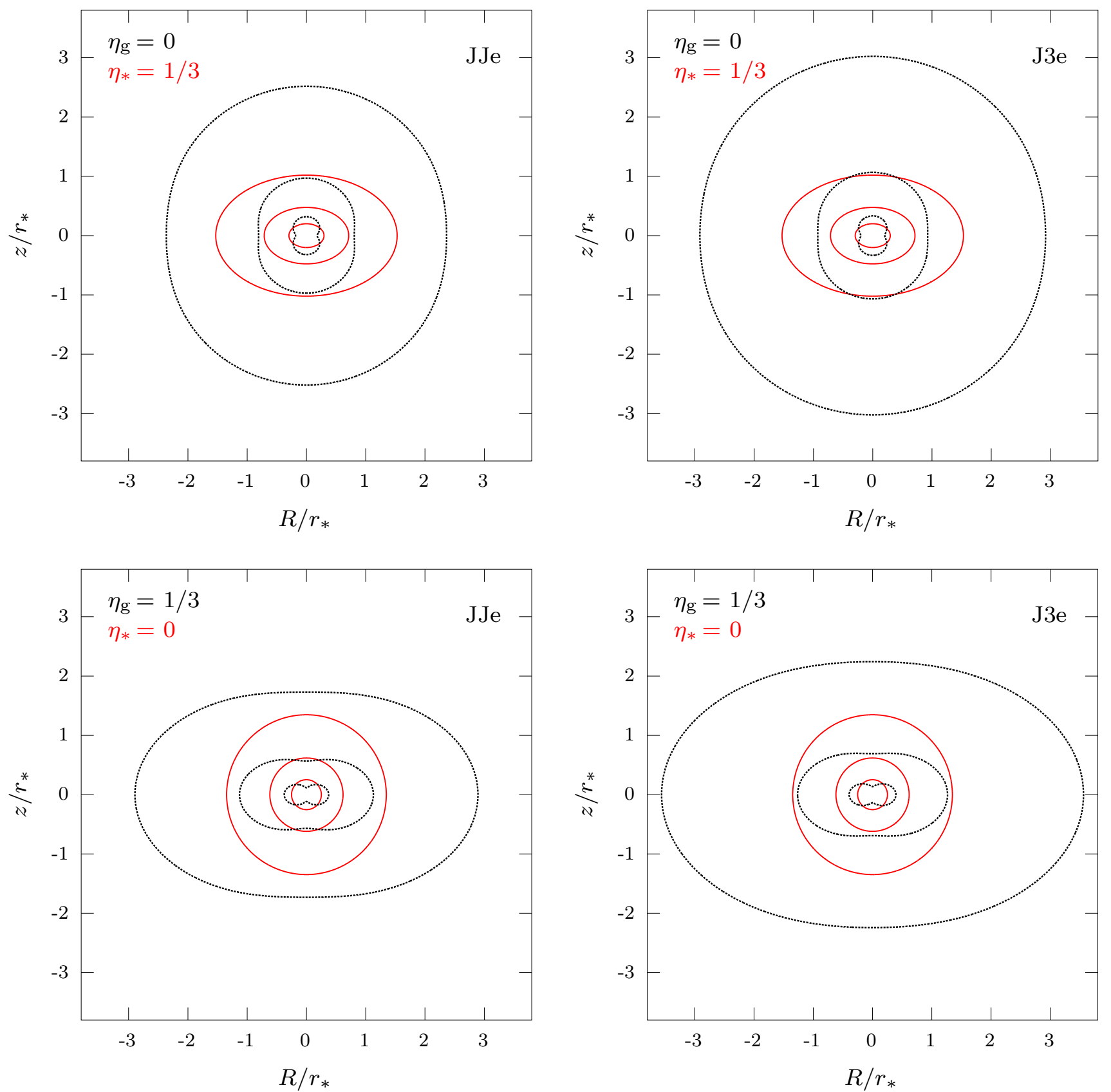

Figure 1. Isodensity contours of the stellar (solid) and DM (dotted) density distributions, for JJe (left) and J3e (right) models. The densities are normalized to $\rho_{\mathrm{n}}$, and the lengths to $r_{*}$. The contours correspond to values of 10,1 , and $10^{-1}$, from inside to outside. Top panels: the stellar distribution is flatter than the total, with $\eta_{*}=1 / 3$, while the galaxy is spherical. Bottom panels: the stellar distribution is spherical, while the total is flatter, with $\eta_{\mathrm{g}}=1 / 3$. Both JJe and J3e models are minimum halo models with $\xi=5$, for which $\mathcal{R}_{\mathrm{m}}=15 / 2$ (see equations 13 and 14).

a function of the parameters is in order. We follow the similar approach already discussed for spherical models in CZ18 and CMP19; of course, the situation is now more complicated, due to the possible different shape of the total and stellar distributions. Quite surprisingly, we find that the discussion can be carried out analytically. We begin by considering the case of two-component ellipsoidal $\gamma$ models (see Dehnen 1993; Tremaine et al. 1994), where the DM density profile is defined as

$$
\rho_{\mathrm{DM}}(R, z)=\frac{(3-\gamma) \rho_{\mathrm{n}} \mathcal{R} \xi}{q_{\mathrm{g}} m_{\mathrm{g}}^{\gamma}\left(\xi+m_{\mathrm{g}}\right)^{4-\gamma}}-\frac{(3-\gamma) \rho_{\mathrm{n}}}{q_{*} m_{*}^{\gamma}\left(1+m_{*}\right)^{4-\gamma}} .
$$

Then, JJe models are the $\gamma=2$ case. In Appendix A we show that the positivity of $\rho_{\mathrm{DM}}$ requires $\mathcal{R} \geq \mathcal{R}_{\mathrm{m}}$, with

$$
\mathcal{R}_{\mathrm{m}}(\mathrm{JJe})= \begin{cases}\frac{q_{\mathrm{g}}}{q_{*}} \max \left(\frac{1}{\xi}, \xi\right), & q_{*} \leq q_{\mathrm{g}}, \\ \frac{q_{*}}{q_{\mathrm{g}}} \max \left(\frac{q_{*}^{2}}{q_{\mathrm{g}}^{2} \xi}, \xi\right), & q_{*} \geq q_{\mathrm{g}} .\end{cases}
$$

Note that, once $q_{*}$ and $q_{\mathrm{g}}$ have been chosen, $\mathcal{R}_{\mathrm{m}}(\mathrm{JJJ}) \geq 1$ for every value of $\xi>0$. A model with $\mathcal{R}=\mathcal{R}_{\mathrm{m}}$ is called a minimum halo 

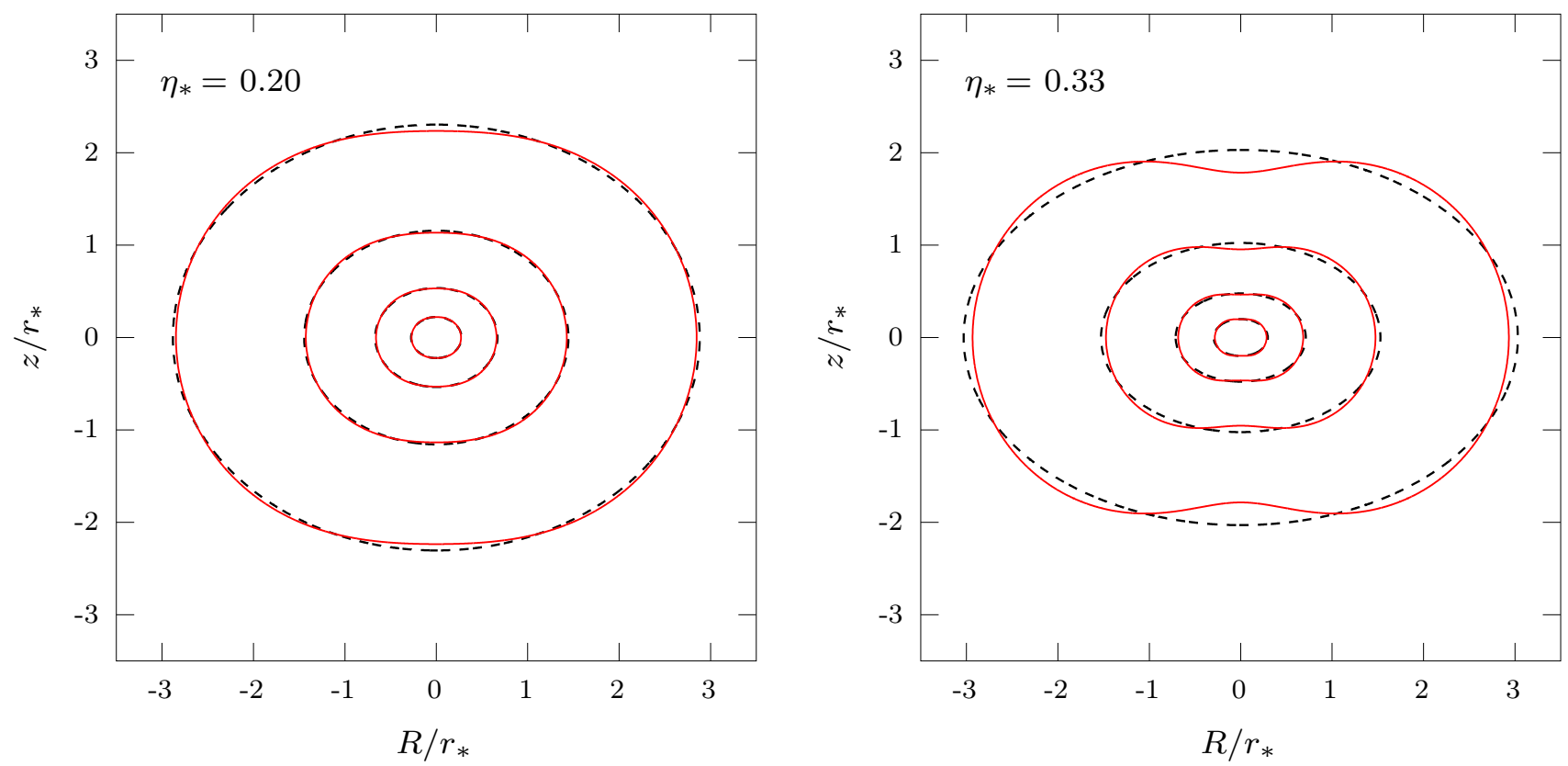

Figure 2. Stellar isodensity contours for two values of $\eta_{*}$, for the true model (dashed), and the homoeoidal expansion (solid); the densities are normalized to $\rho_{\mathrm{n}}$ and the lengths to $r_{*}$. The contours correspond to values of $10,1,10^{-1}, 10^{-2}$, from inside to outside.

model. Clearly, when $q_{\mathrm{g}}=q_{*}$ the positivity conditions reduce to that of spherical JJ models (see CZ18, equation 18).

The positivity condition for the DM component of J3e models is instead given by

$$
\mathcal{R}_{\mathrm{m}}(\mathrm{J} 3 \mathrm{e})=\max \left(\mathcal{R}_{\mathrm{c}}, \mathcal{R}_{0}, \mathcal{R}_{\frac{\pi}{2}}, \mathcal{R}_{\text {int }}\right),
$$

where $\mathcal{R}_{\mathrm{c}}, \mathcal{R}_{0}, \mathcal{R}_{\frac{\pi}{2}}$, and $\mathcal{R}_{\text {int }}$ are given in Appendix A. We may notice that, at variance with the previous case, $\mathcal{R}_{\mathrm{m}}(\mathrm{J} 3 \mathrm{e})$ can be less than unity for some choice of the parameters; this is not surprisingly, since the positivity condition for $\mathrm{J} 3 \mathrm{e}$ models must reduce to that of spherical J3 models for $q_{*}=q_{\mathrm{g}}=0$, which gives $\mathcal{R}_{\mathrm{m}}<1$ when $\xi<1$ (see equation 16 in CMP19).

For illustrative purposes, in Fig. 1 we show the isodensity contours of the stellar and DM density profiles in the meridional plane, for four minimum halo galaxy models. In the top panels the galaxy is spherical $\left(\eta_{\mathrm{g}}=0\right)$, while $\eta_{*}=1 / 3$; in the bottom panels the galaxy is flatter $\left(\eta_{\mathrm{g}}=1 / 3\right)$, while the stellar density is spherical. Note that, as expected, for $\eta_{\mathrm{g}}=0$ the DM distribution is elongated along the $z$-axis, with a prolate-like shape. From the results in Appendix $\mathrm{A}$, one has that a negative DM density is obtained for a total mass below the minimum halo mass. Finally, note how, at any fixed distance from the galactic center (but especially outside $r_{*}$ ), the DM density is larger for J3e than for JJe models.

\subsection{Homoeoidal expansion}

As pointed out in the Introduction, one of the main ideas behind this work is to combine the approach of model construction based on the assignment of the total and stellar profiles (as carried out for spherical models in CMZ09, CZ18, and CMP19) with the homoeoidal expansion technique (see CB05), a methods that allows to describe, in a tractable way, ellipsoidal models in the limit of small flattening. Of course, the models here presented can also be investigated in the case of finite flattenings by using a numerical approach (see Caravita et al. 2020, in preparation), and the comparison of analytical and numerical results is a useful sanity check for both methods. Finally, the present approach, combining the merits of model difference and analytical tractability, is not completely new; in particular, we recall the seminal paper by Evans (1993).

Before presenting the analytical solution of the Jeans equations for the JJe and J3e models (see Sections 3 and 4), we now consider the homoeoidal expansion at fixed mass (the so-called constrained expansion), as a function of the two flattenings $\eta_{*}$ and $\eta_{\mathrm{g}}$. The formulae are obtained from Appendix B, where we also show that, in order to have physically acceptable stellar density, $\eta_{*} \leq 1 / 3$; when considering the total density, instead, equation (B5) shows that $\eta_{\mathrm{g}} \leq 1 / 3$ for JJe models, while $\eta_{\mathrm{g}} \leq 1 / 2$ for J3e models.

The expansion of the total density up to linear terms in the flattenings $\eta_{\mathrm{g}} \rightarrow 0$ reads

$$
\rho_{\mathrm{g}}(R, z)=\rho_{\mathrm{n}} \mathcal{R}\left[\tilde{\rho}_{\mathrm{g} 0}(s)+\eta_{\mathrm{g}} \tilde{\rho}_{\mathrm{g} 1}(s)+\eta_{\mathrm{g}} \tilde{R}^{2} \tilde{\rho}_{\mathrm{g} 2}(s)\right],
$$

where $s=r / r_{*}, \tilde{R}=R / r_{*}$, and the general expression of the three spherical functions is given in Appendix B. As will be discussed in Section 3, there are two different interpretations of the expansion above: one as the linearized expansion of a true ellipsoidal model, and the other as a genuine density distribution with arbitrary values of $\eta_{\mathrm{g}}$, provided that positivity is assured. We shall return to this point.

For JJe and $\mathrm{J} 3 \mathrm{e}$ models, the three dimensionless functions are given by

$$
\tilde{\rho}_{\mathrm{g} i}(\mathrm{JJe})=\left\{\begin{array}{l}
\frac{\xi}{s^{2}(\xi+s)^{2}}, \\
-\frac{\xi(\xi+3 s)}{s^{2}(\xi+s)^{3}}, \\
\frac{2 \xi(\xi+2 s)}{s^{4}(\xi+s)^{3}},
\end{array} \quad \tilde{\rho}_{\mathrm{g} i}(\mathrm{~J} 3 \mathrm{e})=\left\{\begin{array}{l}
\frac{1}{s^{2}(\xi+s)}, \\
-\frac{\xi+2 s}{s^{2}(\xi+s)^{2}}, \\
\frac{2 \xi+3 s}{s^{4}(\xi+s)^{2}},
\end{array}\right.\right.
$$



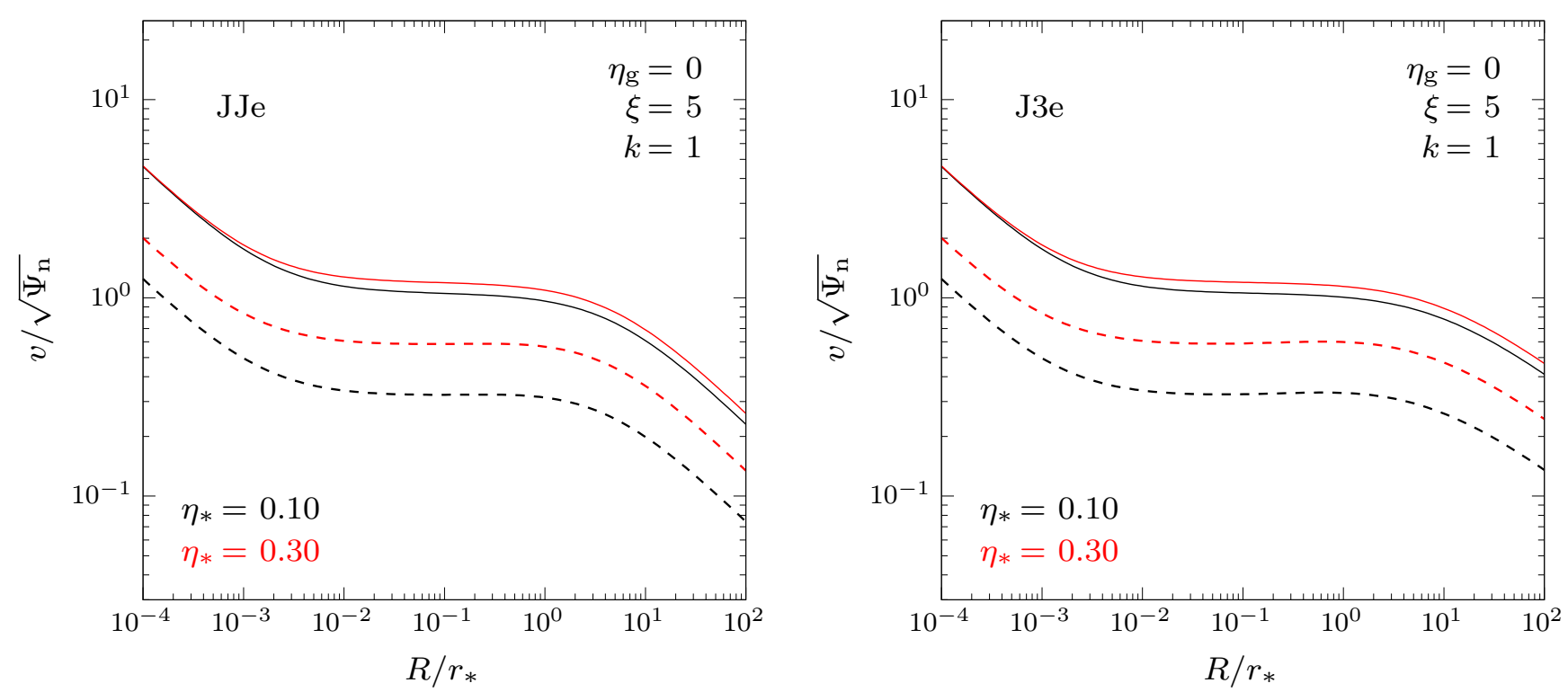

Figure 3. Radial trends of $v_{\mathrm{c}}(R)$ (solid lines) and $\overline{v_{\varphi}}(R, 0)$ (dashed lines), normalized to $\sqrt{\Psi_{\mathrm{n}}}$. Left: JJe models; right: J3e models. Both panels refer to a spherical galaxy, with a central BH with $\mu=0.002$, isotropic orbits (i.e., $k=1$ ), and a minimum halo with $\xi=5$; from equations (13) and (14), $\mathcal{R}_{\mathrm{m}}=50 / 9$ when $\eta_{*}=0.10$, and $\mathcal{R}_{\mathrm{m}}=50 / 7$ when $\eta_{*}=0.30$.

for $i=0,1,2$, from top to bottom, respectively. Note that $\tilde{\rho}_{\mathrm{g} 1}$ is everywhere negative, whereas $\tilde{\rho}_{\mathrm{g} 0}$ and $\tilde{\rho}_{\mathrm{g} 2}$ are positive functions of $s$. Finally, since the stellar distribution is the same for the JJe and $\mathrm{J} 3 \mathrm{e}$ models, and it is of the same family of $\rho_{\mathrm{g}}$ of JJe models, its homoeoidal expansion is

$$
\rho_{*}(\mathrm{JJe}, \mathrm{J} 3 \mathrm{e})=\rho_{\mathrm{n}}\left[\tilde{\rho}_{* 0}(s)+\eta_{*} \tilde{\rho}_{* 1}(s)+\eta_{*} \tilde{R}^{2} \tilde{\rho}_{* 2}(s)\right],
$$

where the functions $\tilde{\rho}_{* i}$ are obtained by setting $\xi=1$ in $\tilde{\rho}_{\mathrm{g} i}(\mathrm{JJe})$. Fig. 2 shows the isodensity contours of the stellar density profile for two different value of $\eta_{*}$. Black dashed lines correspond to the ellipsoidal Jaffe model, described by equation (7), while red solid lines refer to the homoeoidally expanded Jaffe model, provided by equation (15): note how the outermost expanded contours differ from the elliptical shape as $\eta_{*}$ approaches the value $1 / 3$.

The potential associated with equation (15), following Appendix $\mathrm{B}$, can be written as

$$
\Psi_{\mathrm{g}}(R, z)=\Psi_{\mathrm{n}} \mathcal{R}\left[\tilde{\Psi}_{\mathrm{g} 0}(s)+\eta_{\mathrm{g}} \tilde{\Psi}_{\mathrm{g} 1}(s)+\eta_{\mathrm{g}} \tilde{R}^{2} \tilde{\Psi}_{\mathrm{g} 2}(s)\right]
$$

where, as for the density, we recast equation (B10) in terms of $\tilde{R}$. The general integral expressions of the three spherical functions in the equation above are given in Appendix B; for both JJe and $\mathrm{J} 3 \mathrm{e}$ models, all these integrals are elementary, with the normalized densities $\tilde{\rho}(m)=\xi /\left[m^{2}(\xi+m)^{2}\right]$ and $\tilde{\rho}(m)=1 /\left[m^{2}(\xi+m)\right]$, respectively. The final result is

$$
\tilde{\Psi}_{\mathrm{g} i}(\mathrm{JJe})=\left\{\begin{array}{l}
\frac{1}{\xi} \ln \frac{\xi+s}{s} \\
-\frac{s^{2}+2 \xi s+4 \xi^{2}}{3 s^{2}(\xi+s)}+\frac{1}{3 \xi} \ln \frac{\xi+s}{s}+\frac{4 \xi^{2}}{3 s^{3}} \ln \frac{\xi+s}{\xi} \\
\frac{\xi(s+2 \xi)}{s^{4}(\xi+s)}-\frac{2 \xi^{2}}{s^{5}} \ln \frac{\xi+s}{\xi}
\end{array}\right.
$$

and

$$
\tilde{\Psi}_{\mathrm{g} i}(\mathrm{~J} 3 \mathrm{e})=\left\{\begin{array}{l}
\frac{1}{\xi} \ln \frac{\xi+s}{s}+\frac{1}{s} \ln \frac{\xi+s}{\xi} \\
-\frac{s-2 \xi}{3 s^{2}}+\frac{1}{3 \xi} \ln \frac{\xi+s}{s}-\frac{2 \xi^{2}}{3 s^{3}} \ln \frac{\xi+s}{\xi} \\
\frac{s-2 \xi}{2 s^{4}}+\frac{\xi^{2}}{s^{5}} \ln \frac{\xi+s}{\xi}
\end{array}\right.
$$

for $i=0,1,2$, from top to bottom, respectively. As a check, the formulae (16), (19) and (20) have been verified to satisfy the Poisson equation for the dimensionless potential-density pair $\left(\tilde{\Psi}_{\mathrm{g}}, \tilde{\rho}_{\mathrm{g}}\right)$ from the linearization of equation (B12). Finally, the total potential $\Psi_{\mathrm{T}}$ is obtained by adding to $\Psi_{\mathrm{g}}$ the contribution of a central BH of mass $M_{\mathrm{BH}}$, so that the total potential is given by

$$
\Psi_{\mathrm{T}}(R, z)=\Psi_{\mathrm{g}}(R, z)+\frac{\mu \Psi_{\mathrm{n}}}{s} .
$$

A useful quantity to characterize the total potential is the circular velocity in the equatorial plane, given by

$$
v_{\mathrm{c}}^{2}(R)=-R\left(\frac{\partial \Psi_{\mathrm{T}}}{\partial R}\right)_{z=0}=v_{\mathrm{g}}^{2}(R)+v_{\mathrm{BH}}^{2}(R) .
$$

For the homoeoidally expanded models,

$$
v_{\mathrm{c}}^{2}(R)=\Psi_{\mathrm{n}} \mathcal{R}\left[\tilde{v}_{\mathrm{g} 0}^{2}(\tilde{R})+\eta_{\mathrm{g}} \tilde{v}_{\mathrm{g} 1}^{2}(\tilde{R})\right]+\frac{\mu \Psi_{\mathrm{n}}}{\tilde{R}},
$$

where for JJe and J3e models the normalized functions are given by

$$
\tilde{v}_{\mathrm{g} i}^{2}(\mathrm{JJe})=\left\{\begin{array}{l}
\frac{1}{\xi+\tilde{R}}, \\
\frac{\xi(\tilde{R}+2 \xi)}{\tilde{R}^{2}(\xi+\tilde{R})}-\frac{2 \xi^{2}}{\tilde{R}^{3}} \ln \frac{\xi+\tilde{R}}{\xi},
\end{array}\right.
$$


and

$$
\tilde{v}_{\mathrm{g} i}^{2}(\mathrm{~J} 3 \mathrm{e})=\left\{\begin{array}{l}
\frac{1}{\tilde{R}} \ln \frac{\xi+\tilde{R}}{\xi}, \\
\frac{\tilde{R}-2 \xi}{2 \tilde{R}^{2}}+\frac{\xi^{2}}{\tilde{R}^{3}} \ln \frac{\xi+\tilde{R}}{\xi},
\end{array}\right.
$$

for $i=0,1$, from top to bottom, respectively. As expected, galaxy flattening increases the value of $v_{\mathrm{c}}$, because the gravitational field in the equatorial plane, at fixed total mass and major-axis scale-length, becomes stronger for flattened systems. The trend of $v_{\mathrm{c}}(R)$ is shown in Fig. 3 (solid lines) for two minimum halo models with $\xi=5$, $\eta_{\mathrm{g}}=0$, and with $\mu=0.002$ (see Kormendy \& Ho 2013 for this choice of $\mu$ ); black lines show the quite flat case with $\eta_{*}=0.10$, red lines refer to the $\eta_{*}=0.30$ case. Note how, for fixed value of $R$ in the external regions, $v_{\mathrm{c}}(R)$ increases for increasing $\eta_{*}$, due to the dependence of $\mathcal{R}_{\mathrm{m}}$ on $\eta_{*}$; near the center, instead, the $\mathrm{BH}$ contribution is always dominant over that of the galaxy, and so $v_{\mathrm{c}}(R) \propto R^{-1 / 2}$ independently on $\eta_{*}$. Finally, we note that the expansion method allows for simple expressions for the radial epicyclic frequency and vertical epicyclic frequency (see Appendix B).

\subsubsection{Asymptotic behaviour}

For future use we also report the leading term of the density, potential, and circular velocity in the central region and at large radii, as obtained by expansion of the corresponding quantities with $\tilde{R}=$ $s \sin \theta$. At small radii the asymptotic behaviour of density, potential, and circular velocity for JJe models coincides with that of J3e models; we find

$$
\begin{gathered}
\frac{\rho_{\mathrm{g}}}{\rho_{\mathrm{n}}} \sim \mathcal{R} \frac{1+\eta_{\mathrm{g}}\left(1-2 \cos ^{2} \theta\right)}{\xi s^{2}}, \\
\frac{\Psi_{\mathrm{g}}}{\Psi_{\mathrm{n}}} \sim-\mathcal{R} \frac{3+\eta_{\mathrm{g}}}{3 \xi} \ln s, \quad \frac{v_{\mathrm{g}}^{2}}{\Psi_{\mathrm{n}}} \sim \mathcal{R} \frac{3+\eta_{\mathrm{g}}}{3 \xi} .
\end{gathered}
$$

As expected, in absence of a central $\mathrm{BH}, v_{\mathrm{c}}$ reduces to a constant value depending on the models parameters. Instead, in the external regions,

$$
\frac{\rho_{\mathrm{g}}}{\rho_{\mathrm{n}}} \sim \mathcal{R} \times \begin{cases}\xi \frac{1+\eta_{\mathrm{g}}\left(1-4 \cos ^{2} \theta\right)}{s^{4}}, & (\mathrm{JJ} \mathrm{e}), \\ \frac{1+\eta_{\mathrm{g}}\left(1-3 \cos ^{2} \theta\right)}{s^{3}}, & (\mathrm{~J} 3 \mathrm{e}),\end{cases}
$$

$$
\frac{\Psi_{\mathrm{g}}}{\Psi_{\mathrm{n}}} \sim \frac{\mathcal{R}}{s} \times\left\{\begin{array}{ll}
1, & (\mathrm{JJ} \mathrm{e}), \\
\ln s, & (\mathrm{~J} 3 \mathrm{e}),
\end{array} \quad \frac{v_{\mathrm{g}}^{2}}{\Psi_{\mathrm{n}}} \sim \frac{\mathcal{R}}{\tilde{R}} \times \begin{cases}1, & (\mathrm{JJ} \mathrm{e}), \\
\ln \tilde{R}, & (\mathrm{~J} 3 \mathrm{e}) .\end{cases}\right.
$$

Of course, the analogous expressions for the stellar density are obtained by setting $\mathcal{R}=\xi=1$ in equation (26) and in the JJe case of equation (28). Note that at variance with the density, the galaxy potential $\Psi_{\mathrm{g}}$ at large radii is spherical, also for the J3e models with their divergent total mass.

\section{THE JEANS EQUATIONS}

For an axisymmetric density $\rho_{*}(R, z)$ supported by a two-integrals phase-space distribution function $f\left(E, J_{z}\right)$, the Jeans equations for the stellar component are

$$
\begin{gathered}
\frac{\partial \rho_{*} \sigma_{*}^{2}}{\partial z}=\rho_{*} \frac{\partial \Psi_{\mathrm{T}}}{\partial z} \\
\frac{\partial \rho_{*} \sigma_{*}^{2}}{\partial R}-\frac{\rho_{*} \Delta_{*}}{R}=\rho_{*} \frac{\partial \Psi_{\mathrm{T}}}{\partial R}, \quad \Delta_{*} \equiv \overline{v_{\varphi}^{2}}-\sigma_{*}^{2},
\end{gathered}
$$

(see e.g. BT08); $v_{\varphi}$ indicates the azimuthal component of the velocity $\mathbf{v}=\left(v_{R}, v_{\varphi}, v_{z}\right)$, and the "bar-operator" indicates the average value over the velocity-space. These equations are simplified with respect to the general case because for a two-integrals system: (1) the velocity dispersion tensor is aligned with the coordinate system, i.e. the phase-space average of the mixed products of the velocity components vanishes, $\overline{v_{R} v_{z}}=\overline{v_{R} v_{\varphi}}=\overline{v_{\varphi} v_{z}}=0$; (2) the only possible non-zero streaming motion is in the azimuthal direction; (3) the radial and vertical velocity dispersions are equal, i.e. $\sigma_{R}^{2}=\overline{v_{R}^{2}}=\overline{v_{z}^{2}}=\sigma_{z}^{2}$. We define $\sigma_{R}=\sigma_{z} \equiv \sigma_{*}$.

In order to split the azimuthal velocity field in its ordered $\left(\overline{v_{\varphi}}\right)$ and random $\left(\sigma_{\varphi}\right)$ components, we adopt the Satoh (1980) $k$ decomposition

$$
\overline{v_{\varphi}}=k \sqrt{\Delta_{*}},
$$

so that

$$
\sigma_{\varphi}^{2} \equiv \overline{v_{\varphi}^{2}}-{\overline{v_{\varphi}}}^{2}=\sigma_{*}^{2}+\left(1-k^{2}\right) \Delta_{*},
$$

where $k^{2} \leq 1$. This implicitly assumes that the phase-space distribution function depends on $k$, i.e., $f=f\left(E, J_{z} ; k\right)$. The case $k=1$ corresponds to the isotropic rotator, while for $k=0$ no net rotation is present, and all the flattening of $\rho_{*}$ is due to the azimuthal velocity dispersion $\sigma_{\varphi}$. Note that, while in the Satoh decomposition $k$ is independent of position, in principle $k$ can be a function of $(R, z)$, bounded above by the function $k_{\max }(R, z)$, defined by the condition $\sigma_{\varphi}=0$ (see CP96). The $k(R, z)$ formulation can also be used to add counterrotation in a controlled way (see e.g. Negri et al. 2014; see also Caravita et al. 2020).

\subsection{The vertical Jeans equation}

The vertical Jeans equation (30) is integrated at fixed $R$ with the natural boundary condition of a vanishing "pressure" for $z \rightarrow \infty$, so that

$$
\rho_{*} \sigma_{*}^{2}=-\int_{z}^{\infty} \rho_{*} \frac{\partial \Psi_{\mathrm{T}}}{\partial z^{\prime}} d z^{\prime} .
$$

Notice that the integration variable at fixed $R$ can be changed from $z^{\prime}$ to $r^{\prime}$, so that by adopting the expansion (18) the integration acts on spherical coefficients. Due to the relevance of equation (34), a few comments are in order before proceeding to the solution.

The first is that the integral in equation (34) is given by the sum of two contributions: the effect of the galactic potential $\Psi_{\mathrm{g}}$ on the stellar component, and the effect of the central BH. As the BH contribution can be calculated explicitly in terms of elementary functions even considering $\rho_{*}$ in the fully ellipsoidal case, equation (34) can be solved with the homoeoidal approximation.

The second comment concerns the general case of a non-spherical $\Psi_{g}$. As the integral in equation (34) is performed at fixed $R$, it is natural to expand the potential in terms of $\eta_{\mathrm{g}}$, and use the "explicit $-R$ formulation" in equation (18). The three components $\tilde{\Psi}_{\mathrm{g} i}$ are spherically symmetric, so that equation (34) for the fully ellipsoidal stellar density could be again expressed as three integrals over the spherical radius. However, in order to obtain manageable elementary expressions, we also make use of equation (17). 
This leads to a third and final consideration. Due to the linearity of Poisson's equation, the expanded potential-density pairs can be interpreted in two different ways: as a genuinely non spherical system of finite flattening, or as the first order expansion of the ellipsoidal parent galaxy in the limit of vanishing flattening. In the first case, when integrating the Jeans equation, all the terms in the product under the integral should be retained, up to the quadratic order in the flattenings. In the second case, only linear terms in the flattenings are retained. For simplicity, here we limit ourselves to the discussion of the linearized case, and so we consider only the linear terms in $\eta_{*}$ and $\eta_{\mathrm{g}}$. In turn, this choice implies that, in the resulting formulae, only $R^{2}$ terms appear explicitly. Of course, the consideration of quadratic terms in the flattenings does not present special difficulties, only a larger number of computations.

\subsection{The radial Jeans equation}

In principle, once the vertical Jeans equation is solved, no further integration is required because the quantity $\Delta_{*}$ can be obtained from equation (31) by differentiation. However, this straightforward approach may produce formulae that contain non-trivial simplifications, and hide important properties of the solution. These problems are avoided by a very elegant commutator-like formula for the quantity $\Delta_{*}$. For untruncated distributions with vanishing "pressure" at infinity, equation (31) can be recast as a commutator-like integral:

$$
\frac{\rho_{*} \Delta_{*}}{R}=\int_{z}^{\infty}\left(\frac{\partial \Psi_{\mathrm{T}}}{\partial R} \frac{\partial \rho_{*}}{\partial z^{\prime}}-\frac{\partial \Psi_{\mathrm{T}}}{\partial z^{\prime}} \frac{\partial \rho_{*}}{\partial R}\right) d z^{\prime} \equiv\left[\Psi_{\mathrm{T}}, \rho_{*}\right] .
$$

It is not surprising that this relation appears both in Fluid Dynamics (see e.g. Rosseland 1926; Waxman 1978; Barnabè et al. 2006, and references therein), and in Stellar Dynamics (see Hunter 1977), due to the strict relation of the isotropic Jeans equations and hydrodynamic equations. By looking at equation (35), a few important considerations follow. First, for any pair of purely radial functions, the commutator vanishes. Thus, for fully spherical models one has $\Delta_{*}=0$, so that, in the Satoh decomposition, spherical models cannot rotate, and are necessarily isotropic, independently of the value of $k$. In a spherical stellar density, the only non-zero contribution to equation (34) (and so to rotation in the Satoh decomposition) can be produced by a non-spherical dark matter halo, and similarly, in presence of a spherical total potential, rotation can arise only for non-spherical stellar distributions. This is the case of the BH contribution, where the only rotation is due to the departure of the stellar distribution from spherical symmetry.

Moreover, for generic spherically simmetric functions $u$ and $v$, and generic function $f$ of the cylindrical radius, it holds that

$$
[f(R) u(r), v(r)]=\frac{d f}{d R} \int_{r}^{\infty} u\left(r^{\prime}\right) \frac{d v\left(r^{\prime}\right)}{d r^{\prime}} d r^{\prime},
$$

as it can be easily proved by direct computation, and with a final change of integration variable from $z^{\prime}$ to $r^{\prime}$. Therefore, $\Delta_{*}$ in equation (31), at linear order in the flattenings, can be produced only by the effect of the $\Psi_{2}$ term on $\rho_{0}$, and by the $\Psi_{0}$ term on $\rho_{2}$. The resulting $\Delta_{*}$ is proportional to $R^{2}$, so in the Satoh decomposition $\Delta_{*}$ vanishes on the $z$-axis, for sufficiently regular density distributions.

We conclude this Section by noting that the possibility of using the Satoh decomposition depends on the positivity of $\Delta_{*}$, a condition that can be violated for arbitrary choices of the density components. This problem is analogous to the issue encountered in the construction of rotating baroclinic configurations of assigned density distribution in Fluid Dynamics; here, of course, equations (30) and (31) are restricted to the isotropic case, and the velocity dispersion is substituted by the thermodynamic temperature (see Barnabè et al. 2006).

\section{THE SOLUTION}

First, we provide the solution of the vertical Jeans equation, retaining for simplicity only first order terms in the flattenings, as discussed in detail in the previous Section. In full generality we have

$$
\sigma_{*}^{2}=\sigma_{\mathrm{BH}}^{2}+\sigma_{\mathrm{g}}^{2},
$$

where $\sigma_{\mathrm{BH}}$ and $\sigma_{\mathrm{g}}$ represent the contribution of the central $\mathrm{BH}$ and of the galaxy potential to the stellar velocity dispersion $\sigma_{*}$. From the expansion of $\rho_{*}$ it follows that the velocity dispersion profile due to the $\mathrm{BH}$ is given by

$$
\rho_{*} \sigma_{\mathrm{BH}}^{2}=\rho_{\mathrm{n}} \Psi_{\mathrm{n}} \mu\left[A(s)+\eta_{*} B(s)+\eta_{*} \tilde{R}^{2} C(s)\right],
$$

where $A(s), B(s)$, and $C(s)$ are obviously the same for the JJe and $\mathrm{J} 3 \mathrm{e}$ models, and are given in Appendix $\mathrm{C}$. The formualae of the contribution of $\Psi_{\mathrm{g}}$ to the stellar velocity dispersion are more complicated, depending also on the non-spherical component of the galactic potential. At the linear order in the flattenings we have

$$
\begin{aligned}
\rho_{*} \sigma_{\mathrm{g}}^{2} & =\rho_{\mathrm{n}} \Psi_{\mathrm{n}} \mathcal{R}\left[D(s)+\eta_{*} E(s)+\eta_{*} \tilde{R}^{2} F(s)\right. \\
& \left.+\eta_{\mathrm{g}} G(s)+\eta_{\mathrm{g}} \tilde{R}^{2} H(s)\right],
\end{aligned}
$$

and the functions from $D(s)$ to $H(s)$, for JJe and J3e models, are given in Appendix C. Fig. 4 shows a map of $\sigma_{*}$ values in the meridional plane, for minimum halo models with $\xi=5, \eta_{\mathrm{g}}=0$, and $\mu=0.002$. Note the clear elongation of the curves with constant $\sigma_{*}$ along the $z$-axis; this behavior is qualitatively explained by the oblate stellar density shape, to which $\sigma_{*}$ must "compensate", in order for the product $\rho_{*} \sigma_{*}^{2}$ to be roughly spherical (see equation 34, where $\Psi_{\mathrm{T}}$ is spherical).

Second, we evaluate $\Delta_{*}=\Delta_{\mathrm{BH}}+\Delta_{\mathrm{g}}$ from the radial Jeans equation, where $\Delta_{\mathrm{BH}}$ and $\Delta_{\mathrm{g}}$ are the contribution of the $\mathrm{BH}$ and of the galaxy potential, respectively. At the linear order in the flattenings, by using the general considerations in Section 3.2, and in particular equation (35), remarkable identity holds

$$
\rho_{*} \Delta_{\mathrm{BH}}=2 \rho_{\mathrm{n}} \Psi_{\mathrm{n}} \mu \eta_{*} \tilde{R}^{2} C(s),
$$

where $C(s)$ is the same function appearing in equation (38). As anticipated in Section 3.1, it is possible to solve analytically the full homoeoidal problem, but for simplicity here we limit ourselves to the first order expansion. Thus, the galactic contribution to $\Delta_{*}$ is given by

$$
\rho_{*} \Delta_{\mathrm{g}}=2 \rho_{\mathrm{n}} \Psi_{\mathrm{n}} \mathcal{R} \tilde{R}^{2}\left[\eta_{*} F(s)+\eta_{\mathrm{g}} H(s)-\eta_{\mathrm{g}} \tilde{\rho}_{* 0}(s) \tilde{\Psi}_{\mathrm{g} 2}(s)\right],
$$

where $F(s)$ and $H(s)$ are the same functions in equation (39), and $\tilde{\Psi}_{\mathrm{g} 2}(s)$ is given in equations (19) and (20) for JJe and J3e models. Note that, as expected, the two contributions to $\rho_{*} \Delta_{*}$ vanish for $\eta_{*}=\eta_{\mathrm{g}}=0$.

Fig. 5 shows a map of $\sigma_{\varphi}$ values in the meridional plane, for the same minimum halo models of the previous Fig. 4, and in absence of net rotation (i.e., $k=0$ ). In both Figs. 4 and 5 one can note the flatter decline of $\sigma_{*}$ and $\sigma_{\varphi}$, moving outward from the center, for J3e than for JJe models, due to the shallower DM distribution of J3e models (see Fig. 1). 

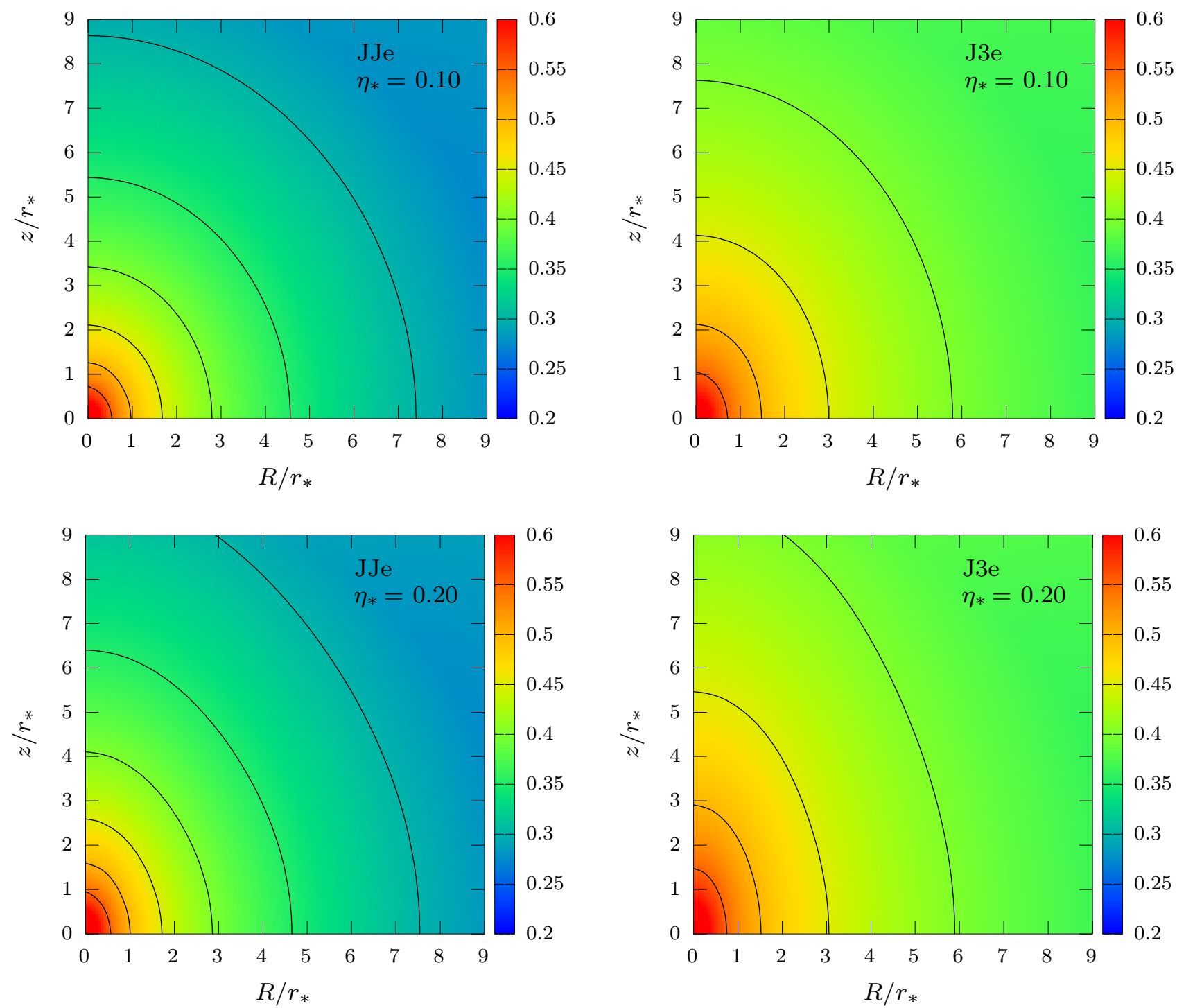

Figure 4. Maps of $\sigma_{*}(R, z)$, normalized to $\sqrt{\Psi_{\mathrm{n}}}$, for two representative minimum halo JJe (left panels) and J3e (right panels) models with $\xi=5$, for a spherical galaxy $\left(\eta_{\mathrm{g}}=0\right)$ and two values of $\eta_{*}$. A central BH with $\mu=0.002$ is also present. The contour lines correspond to values that, starting from 0.55 , decrease with step 0.05 from inside to outside. From equations (13) and (14), $\mathcal{R}_{\mathrm{m}}=50 / 9$ when $\eta_{*}=0.10$, and $\mathcal{R}_{\mathrm{m}}=25 / 4$ when $\eta_{*}=0.20$, for both JJe and J3e models.

Fig. 3 shows $\overline{v_{\varphi}}$ in the equatorial plane, compared with $v_{\mathrm{c}}(R)$, for isotropic $(k=1)$ minimum halo models, with $\xi=5, \eta_{\mathrm{g}}=0$, and $\mu=0.002$, and two shapes for the stellar density $\left(\eta_{*}=0.10\right.$ and 0.30 ). For the same models, but $\eta_{*}=0.10$ and 0.20 , Fig. 6 shows the maps of $\overline{v_{\varphi}}$ in the meridional plane. In both Figs. 3 and 6 the values of $\overline{v_{\varphi}}$ keep larger for the J3e model than for the JJe one, at the same distance from the galactic center, again due to the shallower DM distribution (see Fig. 1).

Finally, we present in Fig. 7 the trends on the equatorial plane of two angular momenta per unit mass, $J_{\mathrm{c}}(R)=R v_{\mathrm{c}}(R)$ and $J_{z}(R)=R \overline{v_{\varphi}}(R, 0)$, for the same models of the Fig. 3. The circular orbit with velocity $v_{\mathrm{c}}$ corresponds to the minimum energy, and thus this figure gives an idea of the radius $R$ where a unit mass ends up in the equatorial plane, after dissipating the maximum possible of its energy, while conserving angular momentum. The practical case here is that of a parcel of gas with a specific angular momentum $J_{z}(R, z)$, that falls on the equatorial plane at some $R$, and then moves inward until it reaches the minimum $R_{\text {in }}$ corresponding to $J_{\mathrm{c}}\left(R_{\text {in }}\right)=J_{z}(R, 0)$. If the gas origin is in stars close to the equatorial plane, and the gas inherits the $J_{z}(R, 0)$ of its parent stars, then it moves inward crossing a radial interval $R-R_{\text {in }}$ given by the condition $J_{\mathrm{c}}\left(R_{\text {in }}\right) \simeq J_{z}(R, 0)$; this interval can be derived directly from Fig. 7. Fig. 8 further illustrates these points: the maps of $J_{z}(R, z)$ give an idea of where gas may end up if falling on the equatorial plane; the colour bars below the maps show the $J_{\mathrm{c}}(R)$ values for a short $R$-range, and allow to link the various $J_{z}(R, 0)$ to the minimum radii $R_{\text {in }}$ that the gas reaches through motions at constant $J_{z}$ but dissipating energy, while on the equatorial plane. For the models in the Figures, gas from the bulk of the galaxies $\left(\simeq 7.5 r_{*}\right)$ is expected to end up within "disks" of just $R \lesssim 2 r_{*}$. 

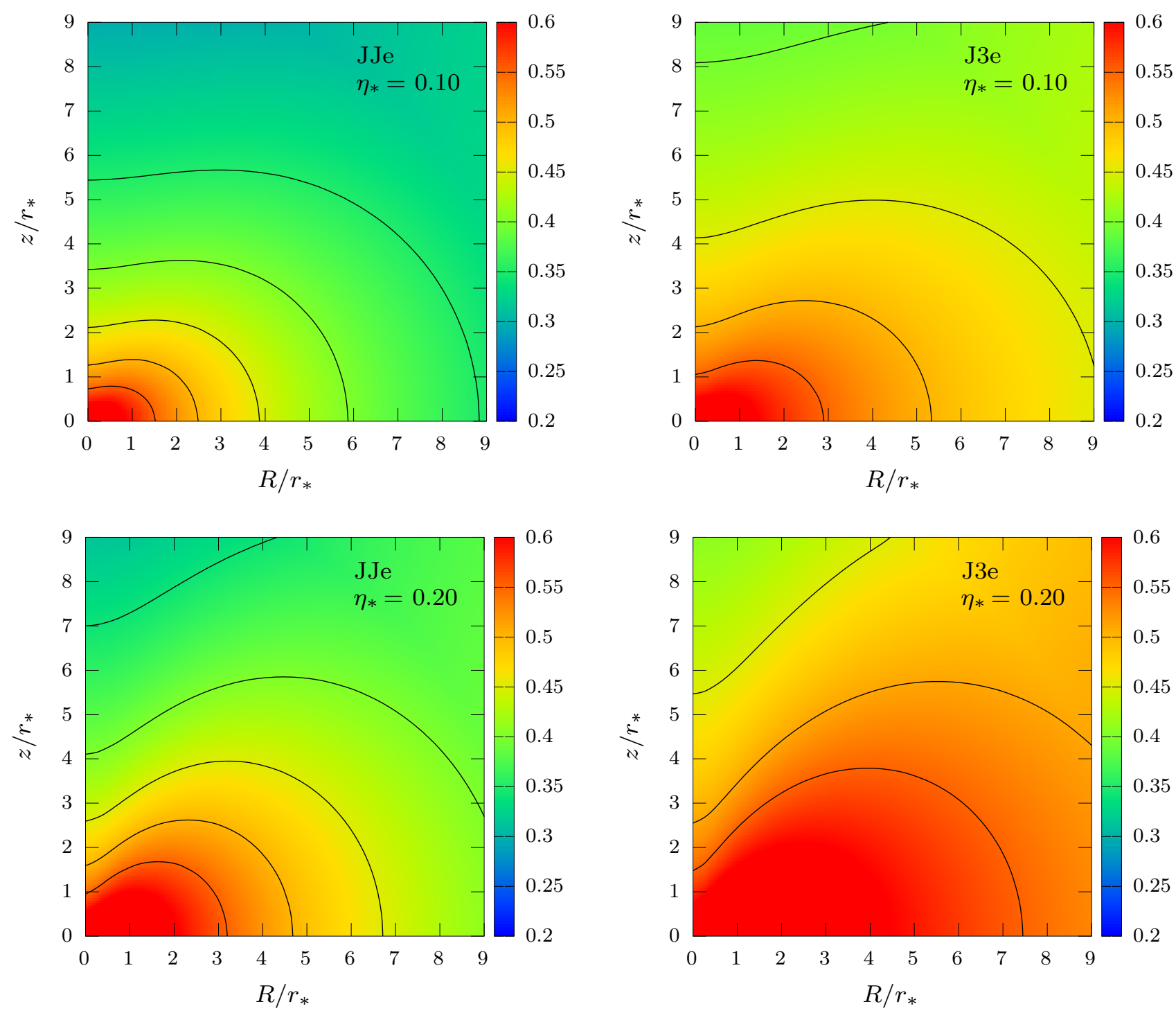

Figure 5. Maps of $\sigma_{\varphi}(R, z)$, normalized to $\sqrt{\Psi_{\mathrm{n}}}$, for the same minimum halo JJe (left panels) and J3e (right panels) models of Fig. 4, in absence of rotation $(k=0)$. The contour lines correspond to values that, starting from 0.55 , decrease with step 0.05 from inside to outside.

\subsection{Asymptotic behavior}

A more quantitative analysis of the effects of the model parameters on the dynamical properties of the stellar component is provided by the asymptotic expansion of the solutions near the center and at large radii.

Near the center (i.e., for $s \rightarrow 0$ ), from Taylor expansion with $\tilde{R}=s \sin \theta$, the asymptotic behaviour of $\rho_{*} \sigma_{*}^{2}$ and $\rho_{*} \Delta_{*}$ for JJe models coincides with that of J3e models. By expanding up to the dominant term of the galaxy contribution we find

$$
\begin{aligned}
\frac{\rho_{*} \sigma_{*}^{2}}{\rho_{\mathrm{n}} \Psi_{\mathrm{n}}} & \sim \mu\left[\frac{5+\eta_{*}\left(1-6 \cos ^{2} \theta\right)}{15 s^{3}}-\frac{2+\eta_{*} \sin ^{2} \theta}{2 s^{2}}\right] \\
& +\mathcal{R} \frac{3\left(1-\eta_{*} \cos ^{2} \theta\right)+\eta_{\mathrm{g}}\left(1+\sin ^{2} \theta\right)}{6 \xi s^{2}},
\end{aligned}
$$

and

$$
\frac{\rho_{*} \Delta_{*}}{\rho_{\mathrm{n}} \Psi_{\mathrm{n}}} \sim \mu\left(\frac{4}{5 s^{3}}-\frac{1}{s^{2}}\right) \eta_{*} \sin ^{2} \theta+\mathcal{R} \frac{\left(3 \eta_{*}-\eta_{\mathrm{g}}\right) \sin ^{2} \theta}{3 \xi s^{2}} .
$$

At large radii (i.e., when $s \rightarrow \infty$ ) we have

$$
\frac{\rho_{*} \sigma_{*}^{2}}{\rho_{\mathrm{n}} \Psi_{\mathrm{n}}} \sim \frac{7-\eta_{*}\left(1+20 \cos ^{2} \theta\right)}{35 s^{5}} \times \begin{cases}\mathcal{R}+\mu, & (\mathrm{JJ} \mathrm{e}), \\ \mathcal{R} \ln s, & (\mathrm{~J} 3 \mathrm{e}),\end{cases}
$$

and

$$
\frac{\rho_{*} \Delta_{*}}{\rho_{\mathrm{n}} \Psi_{\mathrm{n}}} \sim \frac{8 \eta_{*} \sin ^{2} \theta}{7 s^{5}} \times \begin{cases}\mathcal{R}+\mu & (\mathrm{JJ} \mathrm{e}) \\ \mathcal{R} \ln s & (\mathrm{~J} 3 \mathrm{e}) .\end{cases}
$$

The trends above suggest three comments. First, equations (42) and (44) coincide, for $\eta_{*}=\eta_{\mathrm{g}}=0$, with the analogous formulae in CZ18 and CMP19 for the fully isotropic case, as expected. Second, note how the BH mass appears in equation (44) for JJe models, due to the total finite mass, so that the $\sigma_{*}$ is dominated by the monopole term of $\Psi_{\mathrm{g}}$. Of course, the presence of $\mu$ is totally irrelevant for any practical application. For the same reason, $\mu$ does not appear in the case of the $\mathrm{J} 3 \mathrm{e}$ models, which have an infinite mass. Third, the present models exhibit a peculiar behaviour, i.e., near the center 

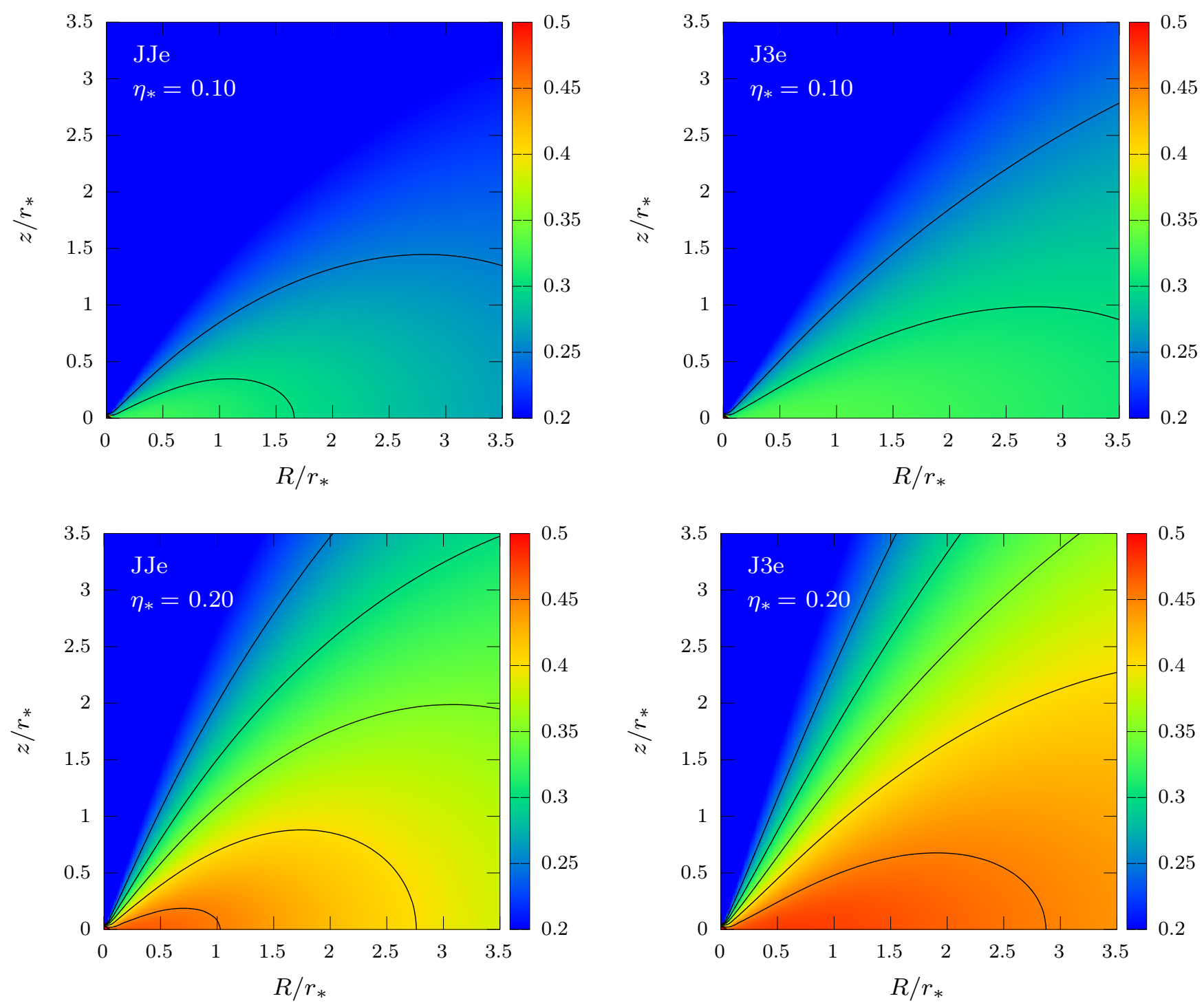

Figure 6. Maps of $\overline{v_{\varphi}}(R, z)$, normalized to $\sqrt{\Psi_{\mathrm{n}}}$, for the same minimum halo JJe (left panels) and J3e (right panels) models of Fig. 4, in the isotropic $(k=1$ ) case. The contour lines correspond to values that, starting from 0.25 , increase with step 0.05 from outside to inside. Note the different ranges of colour and axes values with respect to Fig. 4 and Fig. 5.

the velocity dispersion in the non-spherical case for $\mu=0$ is finite but discontinuous: approaching the center along different $\theta$ directions, one determines different values of the velocity dispersion $\sigma_{*}$. This results from the non-spherical shape of $\rho_{*}$, the central slope of $\rho_{*}$, and the gravitational potential entering the Jeans equations. For example, by using equation (C.3) in Ciotti \& Bertin (2005; see also equation A.4 in Riciputi et al. 2005), it is easy to prove that in the self-gravitating case, the central velocity dispersion for the density profile $1 / m^{\gamma}$ is zero for $0<\gamma<2$, finite discountinuous (as the models in this paper) for $\gamma=2$, and infinite for $\gamma>2$. Instead, $\sigma_{*} \rightarrow \infty$ for generic $\gamma$ in presence of a central $\mathrm{BH}$, while $\sigma_{*}$ is finite discontinuous for generic values of $\gamma$ if the ellipsoid is embedded in the potential of the Singular Isothermal Sphere (Ciotti 2021).

\subsection{Asymmetric Drift}

Asymmetric drift (see e.g. BT08 for a definition) plays some role in the phenomenon of radial gas flows in rotating systems (see Smet
2015). Its computation presents no difficulties in the framework of homoeoidal expansion of our models, thus here we present the basic formulae. We recall that the asymmetric drift (hereafter, AD) in the equatorial plane is defined as $\mathrm{AD}=v_{\mathrm{c}}-\overline{v_{\varphi}}$. In turn, we define the function $\mathcal{D}_{\mathrm{c}} \equiv v_{\mathrm{c}}^{2}-{\overline{v_{\varphi}}}^{2}$, which is of easier evaluation in analytical studies; indeed, for moderate values of the asymmetric drift, one has $\mathrm{AD} \simeq \mathcal{D}_{\mathrm{c}} /\left(2 v_{\mathrm{c}}\right)$. By virtue of equations (31) and (32) we readily have

$$
\rho_{*} \mathcal{D}_{\mathrm{c}}=\left(1-k^{2}\right) \rho_{*} \Delta_{*}-R \frac{\partial \rho_{*} \sigma_{*}^{2}}{\partial R}, \quad(z=0) .
$$

For example, in the isotropic case $(k=1)$, by using equations (38) and (39), a Taylor expansion near the center shows that, at the linear order in the flattenings,

$$
\frac{\mathcal{D}_{\mathrm{c}}}{\Psi_{\mathrm{n}}} \sim \mu\left(\frac{5-4 \eta_{*}}{5 \tilde{R}}-2+\eta_{*}\right)+\mathcal{R} \frac{3\left(1-\eta_{*}\right)+2 \eta_{\mathrm{g}}}{3 \xi} .
$$

In presence of a dominant central $\mathrm{BH}$, one finds $\mathcal{D}_{\mathrm{c}} \propto R^{-1}$ with a nowhere negative proportionality constant. When $\mu=0$, instead, 

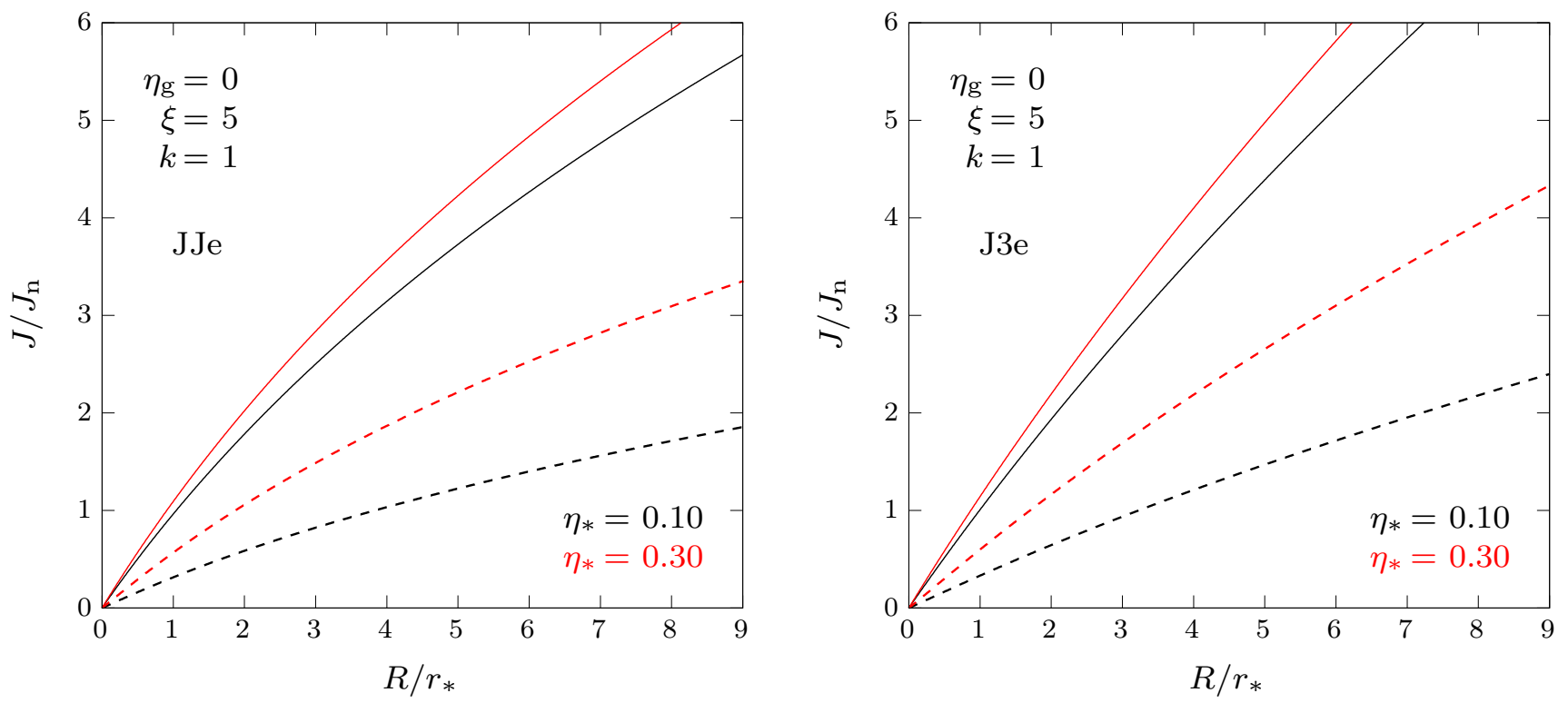

Figure 7. Radial trends of $J_{\mathrm{c}}(R)=R v_{\mathrm{c}}(R)$ (solid lines) and $J_{z}(R, 0)=R \overline{v_{\varphi}}(R, 0)$ (dashed lines), normalized to $J_{\mathrm{n}}=r_{*} \sqrt{\Psi_{\mathrm{n}}}=\sqrt{G M_{*} r_{*}}$, for the same isotropic minimum halo JJe (left) and J3e (right) models of Fig. 3.

$\mathcal{D}_{\mathrm{c}}$ reduces to a constant value, but also in this case $\mathcal{D}_{\mathrm{c}} \geq 0$ for reasonable values of $\eta_{*}$ (which always cannot exceed $1 / 3$ ) and $\eta_{\mathrm{g}}$ (which cannot exceed $1 / 3$ for JJe models, and $1 / 2$ for J3e models).

In the external regions (i.e., when $R \rightarrow \infty$ ), instead, at the leading order we find

$$
\frac{\mathcal{D}_{\mathrm{c}}}{\Psi_{\mathrm{n}}} \sim \frac{7-8 \eta_{*}}{7 \tilde{R}} \times \begin{cases}\mathcal{R}+\mu, & (\mathrm{JJe}), \\ \mathcal{R} \ln \tilde{R}, & (\mathrm{~J} 3 \mathrm{e}) .\end{cases}
$$

By considering the dominant term of the two previous equations, $\mathcal{D}_{\mathrm{c}} \geq 0$ for reasonable values of $\eta_{*}$, as expected. For general values of $k$, it is sufficient to add in equation (46) the expression for $\rho_{*} \Delta_{*}$ (when $z=0$ ) derived in the previous Section.

\section{PROJECTED DYNAMICS}

The projection of a galaxy model on the plane of the sky is an important step in the model construction, needed in order to determine the observational properties of the model itself. In this paper we deal with axisymmetric models, so we need to specify just a single angle $i$ that gives the direction of the line of sight (hereafter, l.o.s.) to the observer. Moreover, the simple functional form of the density and of the intrinsic kinematical fields in the homoeoidal framework leads to further simplifications.

Let our models be described in a Cartesian inertial frame of reference $S_{0}$, with coordinates $(x, y, z)$. In addition, consider a second orthogonal reference system $S^{\prime}$, with coordinates $(X, Y, Z)$, and same origin as $S_{0}$. Due to axisymmetry, and without loss of generality, the relation between the two sets of coordinates is given by

$$
\left(\begin{array}{l}
x \\
y \\
z
\end{array}\right)=\left(\begin{array}{ccc}
\cos i & 0 & \sin i \\
0 & 1 & 0 \\
-\sin i & 0 & \cos i
\end{array}\right)\left(\begin{array}{l}
X \\
Y \\
Z
\end{array}\right)
$$

with the 1.o.s. being directed along $Z$. With this choice, the (transpose) of the unit vector $\mathbf{n}$, from $S_{0}$ to the observer, is $\mathbf{n}=$ $(\sin i, 0, \cos i)$. Moreover, we consider a counter clockwise rotation of an angle $i$ around the $y$-axis, coincident with the $Y$-axis of the observer. In particular, for $i=0$, corresponding to the so-called faceon projection, the 1.o.s. coincides with the $z$-axis, and $(X, Y)=$ $(x, y)$; for $i=\pi / 2$, corresponding to the so-called edge-on projection, the l.o.s. coincides with the $x$-axis, and $(X, Y)=(-z, y)$. In any case, $Y$ is aligned with the major axis of the projection. The distance from the center of the image is indicated by $R=\sqrt{X^{2}+Y^{2}}$.

Accordingly, the projection of the stellar density is given by

$$
\Sigma_{*} \equiv \int_{-\infty}^{\infty} \rho_{*} d Z
$$

where the function $\rho_{*}$ is expressed from equation (49) in terms of vector $\mathbf{X}$ and angle $i$.

The projection of the component along $\mathbf{n}$ of the ordered velocity, called l.o.s. streaming velocity field and indicated with $v_{\mathrm{los}}$, is given by

$$
\Sigma_{*} v_{\mathrm{los}} \equiv \int_{-\infty}^{\infty} \rho_{*} \overline{\mathbf{v}} \cdot \mathbf{n} d Z
$$

where the overline represents the mean over the phace-space; again, as in equation (50), the left hand side of equation (51) depends on $X, Y$, and $i$. Note that, since the streaming motion of stars occurs only in the azimuthal direction, we have $\overline{\mathbf{v}} \cdot \mathbf{n}=-\overline{v_{\varphi}} \sin i \sin \varphi$; as a consequence, $\overline{v_{\varphi}}>0$ when the galaxy rotates in an anti-clockwise sense. In the limit case of a face-on projection, we have no streaming motion.

The projection of the squared velocity reads

$$
\Sigma_{*} v_{\mathrm{p}}^{2} \equiv \int_{-\infty}^{\infty} \rho_{*} \overline{(\mathbf{v} \cdot \mathbf{n})^{2}} d Z
$$

By introducing the velocity dispersion tensor $\sigma_{i j}^{2}$, and expanding 
equation (52), we have $v_{\mathrm{p}}^{2}=\sigma_{\mathrm{p}}^{2}+V_{\mathrm{p}}^{2}$, where ${ }^{1}$

$$
\Sigma_{*} \sigma_{\mathrm{p}}^{2} \equiv \int_{-\infty}^{\infty} \rho_{*} \sigma_{i j}^{2} n_{i} n_{j} d Z, \quad \Sigma_{*} V_{\mathrm{p}}^{2} \equiv \int_{-\infty}^{\infty} \rho_{*}(\overline{\mathbf{v}} \cdot \mathbf{n})^{2} d Z .
$$

Note that, if a net rotation is present, the projected velocity dispersion $\sigma_{\mathrm{p}}^{2}$ is not the observed velocity dispersion $\sigma_{\text {los }}$; the 1.o.s. velocity dispersion, which is related to the broadening of the spectral lines, is instead given by

$$
\Sigma_{*} \sigma_{\mathrm{los}}^{2} \equiv \int_{-\infty}^{\infty} \rho_{*} \overline{\left(\mathbf{v} \cdot \mathbf{n}-v_{\mathrm{los}}\right)^{2}} d Z .
$$

Finally, by combining equations (51), (52), and (54), one has

$$
\sigma_{\mathrm{los}}^{2}=\sigma_{\mathrm{p}}^{2}+V_{\mathrm{p}}^{2}-v_{\mathrm{los}}^{2} \text {. }
$$

In a face-on projection, as $\mathbf{n}$ is always perpendicular to $\overline{\mathbf{v}}$, the previous equation reduces to $\sigma_{\text {los }}=\sigma_{\mathrm{p}}$. Moreover, by adopting the Satoh $k$-decomposition, it is easy to show that the coefficient $k$ appears only in $v_{\text {los }}$; indeed, using equations (32) and (33) one has

$$
\Sigma_{*} v_{\mathrm{p}}^{2}=\int_{-\infty}^{\infty} \rho_{*}\left(\sigma_{*}^{2}+\Delta_{*} \sin ^{2} i \sin ^{2} \varphi\right) d Z,
$$

and, in case of a constant $k$,

$$
\Sigma_{*} v_{\mathrm{los}}=-k \int_{-\infty}^{\infty} \rho_{*} \sqrt{\Delta_{*}} \sin i \sin \varphi d Z
$$

In the more general case of a coordinate-dependent $k$, this parameter would appear inside the integral. Of course, since $\Delta_{*}$ vanishes when $\eta_{*}=\eta_{\mathrm{g}}=0$, spherical models present no streaming motion.

\subsection{Asymptotic behaviour}

The projection integrals in the previous Section in general may be performed only numerically (see Caravita et al. 2020). Here we focus on the asymptotic expansion of the projected fields at small and large radii, amenable to analytical treatment.

From Taylor expansion, the asymptotic behaviour of the projected stellar density, for both JJe and J3e models, can be written as

$$
\frac{\Sigma_{*}}{\Sigma_{\mathrm{n}}} \sim \pi \begin{cases}\frac{\tilde{R}^{2}+\eta_{*} \tilde{Y}^{2} \sin ^{2} i}{\tilde{R}^{3}}, & \tilde{R} \rightarrow 0, \\ \frac{\tilde{R}^{2}+\eta_{*}\left(\tilde{Y}^{2}-2 \tilde{X}^{2}\right) \sin ^{2} i}{2 \tilde{R}^{5}}, & \tilde{R} \rightarrow \infty,\end{cases}
$$

where $\Sigma_{\mathrm{n}} \equiv M_{*} /\left(4 \pi r_{*}^{2}\right)$, and the tilde indicates the normalization with respect to $r_{*}$.

In the face-on case, for which $X=x$ and $Y=y$, we have $\sigma_{i j}^{2} n_{i} n_{j}=\sigma_{*}^{2}$. Again, from the asymptotic analysis of $\rho_{*} \sigma_{*}^{2}$ (see Section 4.1), the behaviour of $\sigma_{\text {los }}$ in the central region is the same for both JJe and J3e models, and it reads

$$
\frac{\Sigma_{*} \sigma_{\mathrm{los}}^{2}}{\Sigma_{\mathrm{n}} \Psi_{\mathrm{n}}} \sim \mu\left[\frac{2\left(5-\eta_{*}\right)}{15 \tilde{R}^{2}}-\frac{\pi\left(4+\eta_{*}\right)}{4 \tilde{R}}\right]+\mathcal{R} \frac{\pi\left(2-\eta_{*}+\eta_{\mathrm{g}}\right)}{4 \xi \tilde{R}} .
$$

Notice that, in absence of the central $\mathrm{BH}, \sigma_{\text {los }}^{2}$ reduces to a nowhere negative constant value for acceptable values of $\eta_{*}$ and $\eta_{\mathrm{g}}$. Very far from the center, instead, at the leading order we have

$$
\frac{\Sigma_{*} \sigma_{\text {los }}^{2}}{\Sigma_{\mathrm{n}} \Psi_{\mathrm{n}}} \sim \frac{4\left(7-5 \eta_{*}\right)}{105 \tilde{R}^{4}} \times \begin{cases}\mathcal{R}+\mu, & (\mathrm{JJe}) \\ \mathcal{R} \ln \tilde{R}, & (\mathrm{~J} 3 \mathrm{e})\end{cases}
$$

\footnotetext{
1 The quantity $v_{\mathrm{p}}$ coincides with $V_{\mathrm{rms}}$ in Cappellari et al. (2013).
}

In the edge-on case, in which $X=-z$ and $Y=y$, the quantities $V_{\mathrm{p}}$ and $v_{\text {los }}$ are not zero. By considering only the leading order terms of equations (42) and (43), and limiting to the lowest in the flattenings, some careful algebra shows that, for $\tilde{R} \rightarrow 0$,

$$
\frac{\Sigma_{*} \sigma_{\mathrm{los}}^{2}}{\Sigma_{\mathrm{n}} \Psi_{\mathrm{n}}} \sim \begin{cases}\mathcal{R} \frac{a_{\mathrm{g}}(\tilde{X}, \tilde{Y})}{\tilde{R}}, & (\mu=0), \\ \mu \frac{a_{\mathrm{BH}}(\tilde{X}, \tilde{Y})}{\tilde{R}^{2}}, & (\mu \neq 0),\end{cases}
$$

and

$$
\frac{\Sigma_{*} v_{\mathrm{los}}}{\Sigma_{\mathrm{n}} \sqrt{\Psi_{\mathrm{n}}}} \sim-k \begin{cases}\sqrt{\mathcal{R} \frac{3 \eta_{*}-\eta_{\mathrm{g}}}{3 \xi}} \frac{2 \tilde{Y}}{\tilde{R}^{2}}, & (\mu=0), \\ \sqrt{\frac{\mu \eta_{*}}{5}} B\left(\frac{1}{2}, \frac{5}{4}\right) \frac{2 \tilde{Y}}{\tilde{R}^{5 / 2}}, & (\mu \neq 0),\end{cases}
$$

where $B(p, q)$ is Euler's complete Beta function. In the external regions, instead,

$$
\frac{\Sigma_{*} \sigma_{\operatorname{los}}^{2}}{\Sigma_{\mathrm{n}} \Psi_{\mathrm{n}}} \sim \frac{4 b(\tilde{X}, \tilde{Y})}{7 \tilde{R}^{4}} \times \begin{cases}\mathcal{R}+\mu, & (\mathrm{JJe}) \\ \mathcal{R} \ln \tilde{R}, & (\mathrm{~J} 3 \mathrm{e})\end{cases}
$$

and

$$
\frac{\Sigma_{*} v_{\mathrm{los}}}{\Sigma_{\mathrm{n}} \sqrt{\Psi_{\mathrm{n}}}} \sim-k \sqrt{\frac{2 \eta_{*}}{7}} B\left(\frac{1}{2}, \frac{9}{4}\right) \frac{2 \tilde{Y}}{\tilde{R}^{9 / 2}} \times\left\{\begin{array}{l}
\sqrt{\mathcal{R}+\mu}, \\
\sqrt{\mathcal{R} \ln \tilde{R}}
\end{array}\right.
$$

the functions $a_{\mathrm{g}}, a_{\mathrm{BH}}$, and $b$, are given in Appendix C3. As expected, equations (59), (60), (61) and (63) reduce, for $\eta_{*}=\eta_{\mathrm{g}}=0$, to the analogous formulae given in CZ18 and CMP19 for the spherical fully isotropic case.

\section{THE VIRIAL THEOREM}

The Virial Theorem (hereafter, VT) provides important information about the global energetics of a galaxy model. Here we focus on the VT of the stellar components:

$$
2 K_{*}=-W_{*} \equiv-W_{* \mathrm{~g}}-W_{* \mathrm{BH}},
$$

where

$$
K_{*}=\frac{1}{2} \int \rho_{*}\left(2 \sigma_{*}^{2}+\overline{v_{\varphi}^{2}}\right) d^{3} \mathbf{x}=\frac{1}{2} \int \rho_{*}\left(3 \sigma_{*}^{2}+\Delta_{*}\right) d^{3} \mathbf{x}
$$

is the total kinetic energy of the stars,

$$
W_{* \mathrm{~g}}=\int \rho_{*} \mathbf{x} \cdot \frac{\partial \Psi_{\mathrm{g}}}{\partial \mathbf{x}} d^{3} \mathbf{x}
$$

is the interaction energy of the stars with the gravitational field of the galaxy (stars plus DM), and finally

$$
W_{* \mathrm{BH}}=\int \rho_{*} \mathbf{x} \cdot \frac{\partial \Psi_{\mathrm{BH}}}{\partial \mathbf{x}} d^{3} \mathbf{x}=U_{* \mathrm{BH}},
$$

where $U_{* \mathrm{BH}}=-\int \rho_{*} \Psi_{\mathrm{BH}} d^{3} \mathbf{x}$ is the gravitational energy of the stars due to the central $\mathrm{BH}$. Note that for a Jaffe density distribution, $W_{* \mathrm{BH}}$ diverges near the origin, so that also the volume integral of $\rho_{*} \sigma_{\mathrm{BH}}^{2}$ diverges, as can be verified by direct integration of equation (38).

In the framework of homoeoidal expansion the integrand in equation (67) can be expressed in simple form. Indeed, with some work ${ }^{2}$,

$$
\begin{aligned}
& { }^{2} \text { From equation (18), simple algebra shows that } \\
& \mathbf{x} \cdot \frac{\partial \Psi_{\mathrm{g}}}{\partial \mathbf{x}}=\Psi_{\mathrm{n}} \mathcal{R}\left[\left(\frac{d \tilde{\Psi}_{\mathrm{g} 0}}{d s}+\eta_{\mathrm{g}} \frac{d \tilde{\Psi}_{\mathrm{g} 1}}{d s}\right) s+\eta_{\mathrm{g}} \tilde{R}^{2}\left(s \frac{d \tilde{\Psi}_{\mathrm{g} 2}}{d s}+2 \tilde{\Psi}_{\mathrm{g} 2}\right)\right] .
\end{aligned}
$$



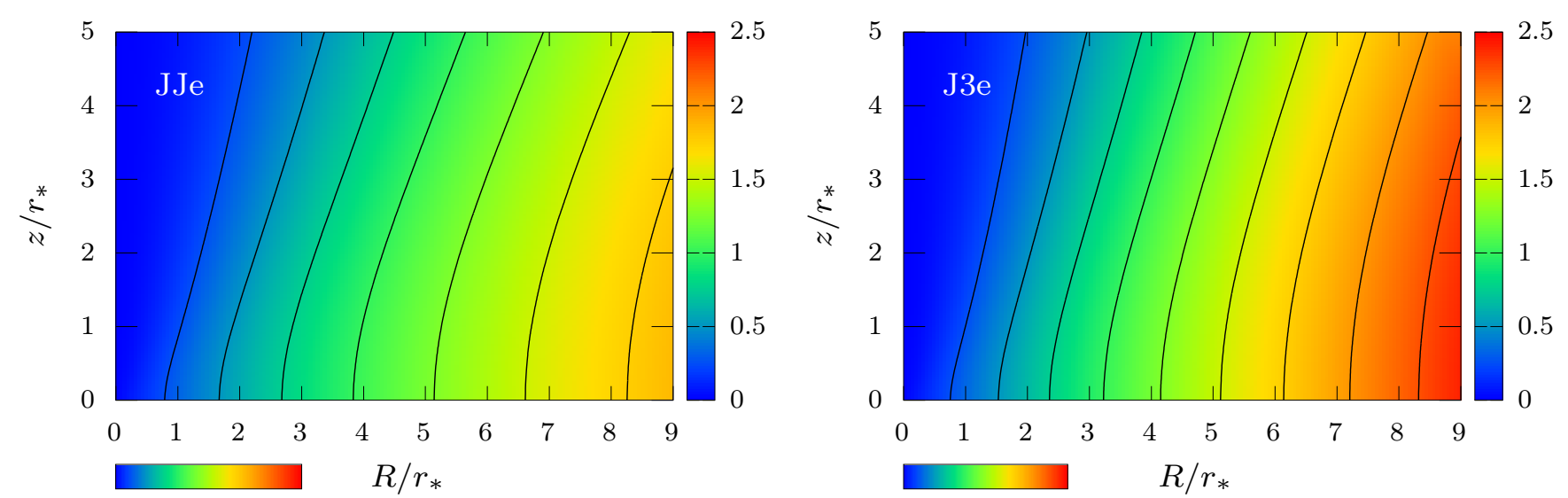

Figure 8. Maps of $J_{z}(R, z)=R \overline{v_{\varphi}}(R, z)$, normalized to $J_{\mathrm{n}}$, for the same isotropic minimum halo models in the two top panels of Fig. 6. The black contour lines correspond to values that, starting from 0.25 , increase with step 0.25 from inside to outside. The bottom colour bars show $J_{\mathrm{c}}(R)=R v_{\mathrm{C}}(R)$, normalized to $J_{\mathrm{n}}$, up to a value of $R$ whereby $J_{\mathrm{c}}(R) / J_{\mathrm{n}}=2.5$ (the discussion at the end of Sect. 4 illustrates the use of such a bar).

after transforming variables to spherical coordinates, an integration over the solid angle shows that, limiting to linear terms in the flattenings,

$$
W_{* \mathrm{~g}}=-U_{\mathrm{n}} \mathcal{R} \times\left(w_{0}+\eta_{*} w_{1}+\eta_{\mathrm{g}} w_{2}\right),
$$

where $U_{\mathrm{n}} \equiv M_{*} \Psi_{\mathrm{n}}$, and

$$
w_{i}=\left\{\begin{array}{l}
-\int_{0}^{\infty} \tilde{\rho}_{* 0} \frac{d \tilde{\Psi}_{\mathrm{g} 0}}{d s} s^{3} d s, \\
-\int_{0}^{\infty}\left(\tilde{\rho}_{* 1}+\frac{2}{3} s^{2} \tilde{\rho}_{* 2}\right) \frac{d \tilde{\Psi}_{\mathrm{g} 0}}{d s} s^{3} d s, \\
-\int_{0}^{\infty} \tilde{\rho}_{* 0} \frac{d}{d s}\left(\tilde{\Psi}_{\mathrm{g} 1}+\frac{2}{3} s^{2} \tilde{\Psi}_{\mathrm{g} 2}\right) s^{3} d s,
\end{array}\right.
$$

for $i=0,1,2$, from top to bottom, respectively.

As well known, in multi-component systems the virial energy $W_{*}$ is not the gravitational energy $U_{*}$ of the stellar component in the total potential. In analogy with the previous discussion, we now express explicitly the different contributions to the potential energy $U_{*}$ of the stellar component. We write

$$
U_{*}=U_{* \mathrm{~g}}+U_{* \mathrm{BH}}=U_{* *}+U_{* \mathrm{DM}}+U_{* \mathrm{BH}} .
$$

In particular,

$$
U_{* \mathrm{~g}}=-\frac{1}{2} \int \rho_{*} \Psi_{*} d^{3} \mathbf{x}-\int \rho_{*} \Psi_{\mathrm{DM}} d^{3} \mathbf{x}=B_{* \mathrm{~g}}-U_{* *},
$$

where

$$
B_{* \mathrm{~g}}=-\int \rho_{*} \Psi_{\mathrm{g}} d^{3} \mathbf{x}
$$

$B_{* \mathrm{~g}}$ is useful in the theory of galactic flows. Indeed, $L_{\mathrm{grav}} \propto\left|B_{* \mathrm{~g}}\right|$, where $L_{\text {grav }}$ is the energy per unit time to be given to the ISM (via, e.g., supernova explosions, or thermalization of the velocity of stellar winds, or AGN feedback) in order to steadily extract the ISM mass injected over the galaxy body in the unit time (e.g., from evolving stars; see Pellegrini 2011, Posacki et al. 2013).

By using the homoeoidal expansion for $\rho_{*}$ and $\Psi_{\mathrm{g}}$, and changing variables to spherical coordinates, an integration over the solid angle shows that, limiting to linear terms in the flattenings,

$$
B_{* \mathrm{~g}}=-U_{\mathrm{n}} \mathcal{R} \times\left(u_{0}+\eta_{*} u_{1}+\eta_{\mathrm{g}} u_{2}\right),
$$

where

$$
u_{i}=\left\{\begin{array}{l}
\int_{0}^{\infty} \tilde{\rho}_{* 0} \tilde{\Psi}_{\mathrm{g} 0} s^{2} d s, \\
\int_{0}^{\infty}\left(\tilde{\rho}_{* 1}+\frac{2}{3} s^{2} \tilde{\rho}_{* 2}\right) \tilde{\Psi}_{\mathrm{g} 0} s^{2} d s, \\
\int_{0}^{\infty} \tilde{\rho}_{* 0}\left(\tilde{\Psi}_{\mathrm{g} 1}+\frac{2}{3} s^{2} \tilde{\Psi}_{\mathrm{g} 2}\right) s^{2} d s,
\end{array}\right.
$$

for $i=0,1,2$, from top to bottom, respectively.

\subsection{The Virial Theorem for JJe models}

For the JJe models the quantities $w_{i}$ in equation (70) can be easily computed as

$$
w_{i}(\mathrm{JJe})=\left\{\begin{array}{l}
\frac{\xi-1-\ln \xi}{(\xi-1)^{2}} \\
\frac{2(1-\xi)+(\xi+1) \ln \xi}{3(\xi-1)^{3}} \\
\frac{\xi^{2}-1-2 \xi \ln \xi}{3(\xi-1)^{3}}
\end{array}\right.
$$

and, for $\xi=1$,

$$
w_{0}=\frac{1}{2}, \quad w_{1}=\frac{1}{18}, \quad w_{2}=\frac{1}{9} .
$$

A few comments are in order. First, in case of $\eta_{*}=\eta_{\mathrm{g}}=0$ the function $W_{* \mathrm{~g}}$ reduces to that of spherical models already given in CZ18. Second, for $\xi \rightarrow \infty$ the contribution to $W_{* g}$ in equation (69) due to the function $w_{1}$ is subdominant with respect those of $w_{0}$ and $w_{2}$, and we get

$$
W_{* \mathrm{~g}} \sim-U_{\mathrm{n}} \mathcal{R} \frac{3+\eta_{\mathrm{g}}}{3 \xi} .
$$

In the minimum halo case, form equation (13) it follows that $W_{* \mathrm{~g}}$ tends to a finite value depending only on the two flattenings $\eta_{*}$ 

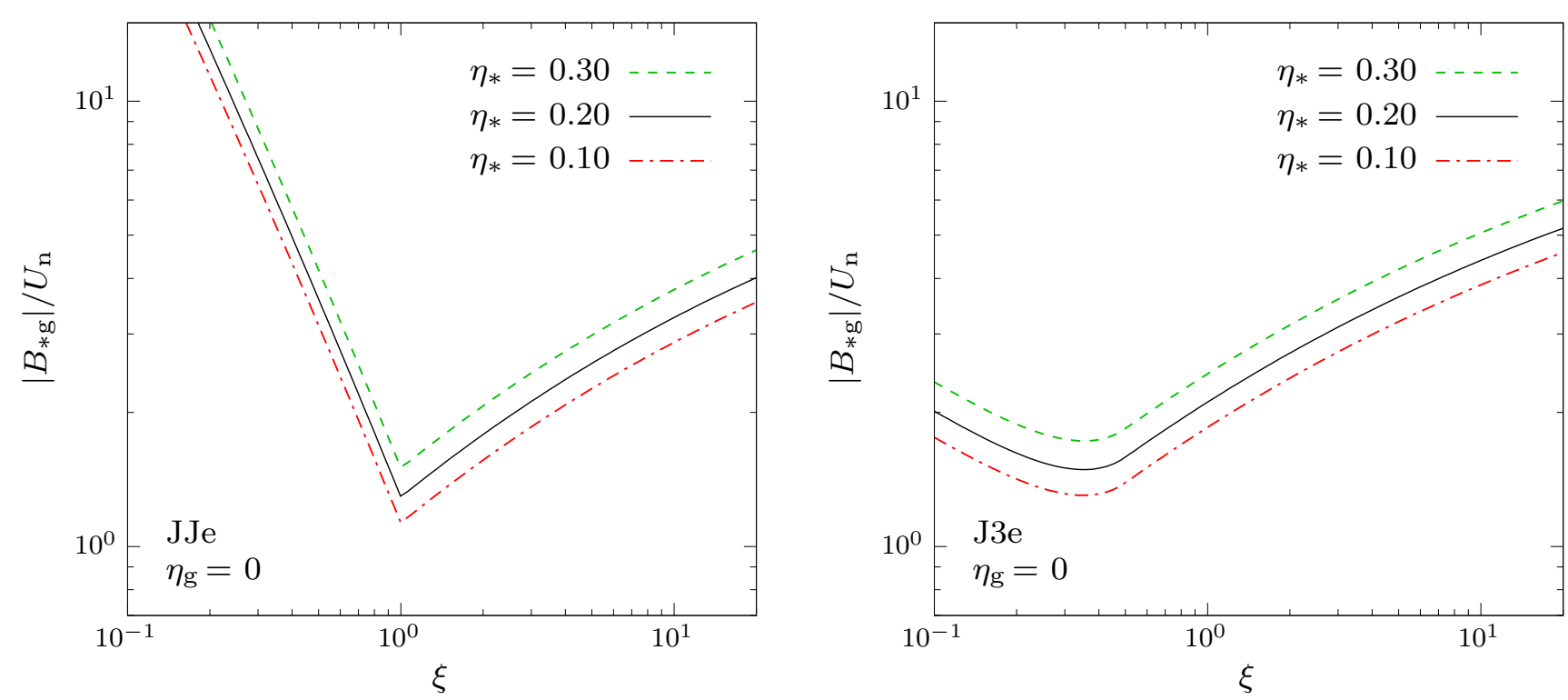

Figure 9. Absolute value of the energy $B_{* \mathrm{~g}}$, normalized to $U_{\mathrm{n}} \equiv \Psi_{\mathrm{n}} M_{*}=G M_{*}^{2} / r_{*}$, as a function of $\xi$, for three representative minimum halo models, for a spherical galaxy without a central BH (i.e., $\mu=0$ ). Left: JJe models; right: J3e models. Notice how for large values of $\xi$ the curves in the two panels have similar behaviour. It can be proved that for $\xi \rightarrow \infty$ they become identical (see Section 6).

and $\eta_{\mathrm{g}}$. The third comment concerns the contribution of the DM to $W_{* \mathrm{~g}}=W_{* *}+W_{* \mathrm{DM}}$ (where $W_{* *}$ is due to the self-interaction of the stellar distribution, and $W_{* \mathrm{DM}}$ to the effect of the DM halo). From equation (69) we have the estimate of $W_{* \mathrm{~g}}$ for small flattenings of the total and stellar distributions. As the total density in JJe models is a Jaffe ellipsoidal model as the stellar one, it is obvious that equation (69) can be used also to estimate $W_{* *}$ for small flattenings, just by considering in it $\mathcal{R}=1, \xi=1$, and $\eta_{\mathrm{g}}=\eta_{*}$, so that $W_{* \mathrm{DM}}=W_{* \mathrm{~g}}-W_{* *}$.

For what concerns the function $B_{* \mathrm{~g}}$, from equation (75) we find

$$
u_{i}(\mathrm{JJe})=\left\{\begin{array}{l}
\frac{\ln \xi}{\xi-1} \\
\frac{\xi-1-\ln \xi}{3(\xi-1)^{2}} \\
\frac{1-\xi+\xi \ln \xi}{3(\xi-1)^{2}}
\end{array}\right.
$$

where, for $\xi=1$,

$$
u_{0}=1, \quad u_{1}=\frac{1}{6}, \quad u_{2}=\frac{1}{6} .
$$

As for $W_{* \mathrm{~g}}$, in the limit case of $\eta_{*}=\eta_{\mathrm{g}}=0$ also the function $B_{* \mathrm{~g}}$ coincides with that found in CZ18. For large values of $\xi$ (i.e., $\xi \rightarrow$ $\infty)$, also in case of $B_{* \mathrm{~g}}$ the spherical term and that due to the total distribution dominate over the stellar one; the resulting behaviour reads

$$
B_{* \mathrm{~g}} \sim-U_{\mathrm{n}} \mathcal{R} \frac{3+\eta_{\mathrm{g}}}{3 \xi} \ln \xi \sim W_{* \mathrm{~g}} \ln \xi .
$$

Therefore, at variance with $W_{* \mathrm{~g}}$, the quantity $B_{* \mathrm{~g}}$ diverges for a DM halo much more extended than the stellar distribution. Note that $U_{* *}$ can also be obtained, for JJe models, not only from $W_{* \mathrm{~g}}$, but also from $B_{* \mathrm{~g}}$, evaluating $B_{* \mathrm{~g}} / 2$ with $\mathcal{R}=1, \xi=1$, and $\eta_{\mathrm{g}}=\eta_{*}$. Once $U_{* *}$ is obtained, the evaluation of $U_{* \mathrm{DM}}$ through $B_{* \mathrm{~g}}$ is trivial, since $U_{* \mathrm{DM}}=B_{* \mathrm{~g}}-2 U_{* *}$. Fig. 9 shows the trend of $\left|B_{* \mathrm{~g}}\right|$ for a spherical galaxy, as a function of $\xi$, for different stellar flattenings.

\subsection{The Virial Theorem for J3e models}

The quantities $w_{0}, w_{1}$ and $w_{2}$ are given by

$$
w_{i}(\mathrm{~J} 3 \mathrm{e})=\left\{\begin{array}{l}
\mathcal{H}(\xi, 0)-\frac{\ln \xi}{\xi-1}, \\
\frac{1}{3} \mathcal{H}(\xi, 0)-\frac{\xi-1+(\xi-2) \ln \xi}{3(\xi-1)^{2}} \\
\frac{\xi-1-\ln \xi}{3(\xi-1)^{2}}
\end{array}\right.
$$

where the function $\mathcal{H}(\xi, s)$ is defined as

$$
\mathcal{H}(\xi, s) \equiv \int_{s}^{\infty} \ln \left(1+\frac{1}{t}\right) \frac{d t}{\xi+t},
$$

and it can be expressed in terms of the dilogarithm function (for more details, see equation C1 in CMP19). In particular, for $\xi=1$,

$$
w_{0}=\frac{\pi^{2}}{6}-1, \quad w_{1}=\frac{\pi^{2}}{18}-\frac{1}{2}, \quad w_{2}=\frac{1}{6} .
$$

In analogy with equation (79), for J3e models the function $B_{* g}$ is determined by

$$
u_{i}(\mathrm{~J} 3 \mathrm{e})=\left\{\begin{array}{l}
\mathcal{H}(\xi, 0), \\
\frac{1}{3} \mathcal{H}(\xi, 0)-\frac{\ln \xi}{3(\xi-1)}, \\
\frac{\ln \xi}{3(\xi-1)},
\end{array}\right.
$$

where, for $\xi=1$,

$$
u_{0}=\frac{\pi^{2}}{6}, \quad u_{1}=\frac{\pi^{2}}{18}-\frac{1}{3}, \quad u_{2}=\frac{1}{3} .
$$

For $\eta_{*}=\eta_{\mathrm{g}}=0$ the quantities $W_{* \mathrm{~g}}$ and $B_{* \mathrm{~g}}$ coincide with that found in CMP19. Moreover, when considering large values of $\xi$, a simple expansion shows that the asymptotic behaviours of $W_{* \mathrm{~g}}$ and $B_{* \mathrm{~g}}$ are identical to that derived in case of JJe models (see Fig. 
9): a qualitative explanation is that in both cases the Jaffe stellar distribution, for $\xi \rightarrow \infty$, is embedded in what we call "singular isothermal ellipsoid".

\section{DISCUSSION AND CONCLUSIONS}

In this paper we present two new families of two-component axially symmetrical galaxy models: the ellipsoidal generalization of the spherical JJ and J3 models, introduced in CZ18 and CMP19, respectively. In both these new families the stellar density follows an ellipsoidal Jaffe profile; then, in the JJe models the total density is described by another ellipsoidal Jaffe law, in the J3e models the total density is such that its difference with the stellar density (e.g. the resulting DM halo) can be made similar to an ellipsoidal NFW model. In both families the total density has a different flattening and scale length with respect to the stellar density. Finally, a BH is also added at the center. The JJe and J3e models are fully determined once the stellar mass $\left(M_{*}\right)$ and the scale length of the stellar density $\left(r_{*}\right)$ are assigned, together with the total-to-stellar scale length ratio $(\xi)$, the total-to-stellar density ratio $(\mathcal{R})$, the flattening of the stellar profile $\left(\eta_{*}\right)$, the flattening of the total profile $\left(\eta_{\mathrm{g}}\right)$, and finally a BH-to-stellar mass ratio $(\mu)$.

One of the main advantages of the JJe and $\mathrm{J} 3 \mathrm{e}$ models is that, thanks to a homoeoidal expansion to the first order adopted in this paper, an analytical treatment of several quantities of interest in theoretical and observational works is possible, as detailed below.

(i) The constraints on $\mathcal{R}$ and $\xi$ to assure the positivity of the DM halo density profile are derived analytically. For a given $\xi$, the model with the minimum value $\mathcal{R}_{\mathrm{m}}$ allowed for $\mathcal{R}$ is called minimum halo model. A general method to discuss the positivity of the DM distribution, defined as the difference between two arbitrary ellipsoidal distributions, is also presented in the Appendix.

(ii) We expand the density and potential profiles for a small deviation from spherical symmetry by adopting the so-called homoeoidal expansion method (see CB05). The derived analytical expressions correspond to positive densities if $\eta_{*} \leq 1 / 3$, and $\eta_{\mathrm{g}} \leq$ $1 / 3(\mathrm{JJe})$ or $\eta_{\mathrm{g}} \leq 1 / 2(\mathrm{~J} 3 \mathrm{e})$. The circular velocity $v_{\mathrm{c}}$ in the equatorial plane $(z=0)$ is also obtained.

(iii) Using the homoeoidal expansions, we analytically solve the two-integral Jeans equations, where the Satoh (1980) $k$ decomposition is adopted to split the azimuthal velocity field in its ordered $\left(\overline{v_{\varphi}}\right)$ and random $\left(\sigma_{\varphi}\right)$ components. The solutions are given at their first order terms in the flattening; the asymptotic expansion near the center and in the outer regions are presented, and maps of $\sigma_{*}, \sigma_{\varphi}$ and $\overline{v_{\varphi}}$ in the meridional plane for a representative galaxy are also shown. The $\sigma_{*}$ contour lines are elongated along the $z$-axis. For fixed distance from the galactic center, the values of $\sigma_{*}, \sigma_{\varphi}$ and $\overline{v_{\varphi}}$ keep larger for the J3e model than for the JJe one, due to the broader DM distribution of the former models. Finally, the link between the angular momentum $J_{z}(R, z)$ away from the equatorial plane, and that on the plane $J_{\mathrm{c}}(R)$, is briefly presented; this link is useful to consider for problems involving infalling gas that conserves its angular momentum.

(iv) Finally, the analytical expressions for the quantities entering the Virial Theorem, such as the interaction energy and the potential energies, are derived as a function of the model parameters.

The JJe and J3e models represent a significant improvement over the spherical counterparts discussed in CZ18 and CMP19: they provide a more advanced modelling of the dynamics of elliptical galaxies, while still keeping realistic mass distributions, as are the Jaffe or the NFW laws. The analytical formulation of all their dynamical properties (e.g. kinematical quantities and virial energies) can be used to understand what is the effect of the various parameters (flattening, scale lengths, mass ratio, rotational support) in determining these properties. The analytical expressions are also useful when realistic axially symmetric two-component galaxy models are needed, for example to be given in input in numerical simulations, as those reproducing galactic flows (see e.g. Gan et al. 2019).

\section{ACKNOWLEDGEMENTS}

We thanks Caterina Caravita, Zhaoming Gan, and Federico Marinacci for careful and independent numerical checks of several formulae. The anonymous referee is warmly thanked for several suggestions that considerably improved the paper.

\section{DATA AVAILABILITY}

No datasets were generated or analysed in support of this research.

\section{REFERENCES}

Barnabè M., Ciotti L., Fraternali F., Sancisi S., 2006, A\&A, 446, 61 Bertin G., 2000, Dynamics of Galaxies, Cambridge University Press Binney J., Tremaine S., 2008, Galactic Dynamics, 2nd Ed., Princeton University Press, Princeton (BT08)

Cappellari M. et al., 2013, MNRAS, 432, 1862

Caravita C., Ciotti L., Pellegrini S., 2020 (in preparation)

Chandrasekhar S., 1969, Ellipsoidal Figures of Equilibrium, Yale University Press, New Haven

Ciotti L., 2021, Introduction to Stellar Dynamics, Cambridge University Press (in press)

Ciotti L., Bertin G., 2005, A\&A, 437, 419 (CB05)

Ciotti L., Morganti L., de Zeeuw P. T. 2009, MNRAS, 393, 491 (CMZ09)

Ciotti L., Mancino A., Pellegrini S., 2019, MNRAS, 490, 2656 (CMP19)

Ciotti L., Pellegrini S., 1996, MNRAS, 279, 240 (CP96)

Ciotti L., Ziaee Lorzad A., 2018, MNRAS, 473, 5476 (CZ18)

Dehnen W., 1993, MNRAS, 265, 250

Evans N. W., 1993, MNRAS, 260, 191

Gan Z., Ciotti L., Ostriker J. P., Yuan F., 2019, ApJ, 872, 167

Hunter C., 1977, AJ, 82, 271

Jaffe W., 1983, MNRAS, 202, 995

Kellogg O. D., 1967, Foundation of Potential Theory, Springer-Verlag, New York

Kormendy J., Ho L. C., 2013, ARA\&A, 51, 511

Magorrian J. et al. 1998, AJ, 115, 2285

Navarro J. F., Frenk C. S., White S. D. M., 1997, ApJ, 490, 493 (NFW)

Negri A., Posacki S., Pellegrini S., Ciotti L., 2014, MNRAS, 445, 1351

Pellegrini S., 2011, ApJ, 738, 57

Posacki S., Pellegrini S., Ciotti L., 2013, MNRAS, 433, 2259

Riciputi A., Lanzoni B., Bonoli S., Ciotti L., 2005, A\&A, 443, 133

Rosseland S., 1926, ApJ, 63, 342

Satoh C., 1980, PASJ, 32, 41

Smet C. O., Posacki S., Ciotti L., 2015, MNRAS, 448, 2921

Tremaine S. et al., 1994, AJ, 107, 634

Waxman A. M., 1978, ApJ, 222, 61

\section{APPENDIX A: POSITIVITY OF THE DM DISTRIBUTION}

In order to discuss the positivity of the DM distribution of JJe and $\mathrm{J} 3 \mathrm{e}$ models, it is convenient to set up the problem in the more general 
case of two arbitrary ellipsoidal distributions, and then to specialized the results to the specific cases. Let

$$
\rho_{*}=E_{*}\left(m_{*}\right), \quad \rho_{\mathrm{g}}=\mathcal{R} E_{\mathrm{g}}\left(m_{\mathrm{g}}\right),
$$

be the stellar and total density distributions, with $E_{*}$ and $E_{\mathrm{g}}$ the arbitrary functions describing the profiles, where $m_{*}$ and $m_{\mathrm{g}}$ are defined in Section 2. We change variables from $(R, z)$ to $(r \sin \theta, r \cos \theta)$, so that

$$
m_{*}=s \Omega_{*}, \quad m_{\mathrm{g}}=s \Omega_{\mathrm{g}}, \quad s=\frac{r}{r_{*}},
$$

and

$$
\Omega_{*}^{2}=\sin ^{2} \theta+\frac{\cos ^{2} \theta}{q_{*}^{2}}, \quad \Omega_{\mathrm{g}}^{2}=\sin ^{2} \theta+\frac{\cos ^{2} \theta}{q_{\mathrm{g}}^{2}} .
$$

The positivity condition for $\rho_{\mathrm{DM}}=\rho_{\mathrm{g}}-\rho_{*}$ becomes

$$
\mathcal{R} \geq \mathcal{R}_{\mathrm{m}}=\sup _{\mathcal{I}} \mathcal{F}(s, \theta), \quad \mathcal{F}(s, \theta)=\frac{E_{*}\left(m_{*}\right)}{E_{\mathrm{g}}\left(m_{\mathrm{g}}\right)},
$$

where, from Fig. A1, $\mathcal{I} \equiv\{(s, \theta) \mid s \geq 0,0 \leq \theta \leq \pi / 2\}$ : we restrict to values of $\theta$ between 0 and $\pi / 2$ since $\mathcal{F}(s, \pi-\theta)=\mathcal{F}(s, \theta)$. A DM halo with $\mathcal{R}=\mathcal{R}_{\mathrm{m}}$ is called a minumim halo: clearly, if $\mathcal{R}$ decreases slightly below $\mathcal{R}_{\mathrm{m}}$, the DM density becomes first negative at the position where $\mathcal{F}(s, \theta)=\mathcal{R}_{\mathrm{m}}$. It follows that

$$
\mathcal{R}_{\mathrm{m}}=\max \left(\mathcal{R}_{\mathrm{c}}, \mathcal{R}_{\infty}, \mathcal{R}_{0}, \mathcal{R}_{\frac{\pi}{2}}, \mathcal{R}_{\text {int }}\right),
$$

where the values in parentheses are the $\sup \mathcal{F}(s, \theta)$ over the corresponding regions in Fig. A1. Geometrically, $\sup \mathcal{F}(s, \theta)$ can be located only at the center, at infinity, on the equatorial plane, along the symmetry axis, or in the interior.

We first show that for $q_{*} \neq q_{\mathrm{g}}$ the function $\mathcal{F}$ has no critical points in $\operatorname{int}(\mathcal{I})$. Indeed, a simple computation shows that its gradient can vanish when

$$
\left\{\begin{array}{l}
\Omega_{*} \frac{d E_{*}}{d m_{*}} E_{\mathrm{g}}=\Omega_{\mathrm{g}} \frac{d E_{\mathrm{g}}}{d m_{\mathrm{g}}} E_{*}, \\
\frac{d \Omega_{*}}{d \theta} \frac{d E_{*}}{d m_{*}} E_{\mathrm{g}}=\frac{d \Omega_{\mathrm{g}}}{d \theta} \frac{d E_{\mathrm{g}}}{d m_{\mathrm{g}}} E_{*},
\end{array}\right.
$$

where the first equation corresponds to $\partial \mathcal{F} / \partial s=0$, and the second to $\partial \mathcal{F} / \partial \theta=0$. The proof proceeds as follows. If the first equation is not satisfied in $\operatorname{int}(\mathcal{I})$, there is nothing to prove. So, let us assume that the first identity is satisfied somewhere in $\operatorname{int}(\mathcal{I})$. Then, for non-negative and monotonically decreasing density distributions, the second equation reduces to

$$
\frac{d \Omega_{*}}{d \theta} \Omega_{\mathrm{g}}=\Omega_{*} \frac{d \Omega_{\mathrm{g}}}{d \theta} ;
$$

however, it is trivial to show that, for $q_{*} \neq q_{\mathrm{g}}$, there are no solutions for $0<\theta<\pi / 2$. We are left with the case $q_{*}=q_{\mathrm{g}}$. In this circumstance, the two equations of the system (A6) become coincident. As a consequence, $\mathcal{R}_{\text {int }}$ must be determined by solving the equation $\partial \mathcal{F} / \partial s=0$, and imposing the condition $q_{*}=q_{\mathrm{g}}$. In particular, we note that in the special case $q_{*}=q_{\mathrm{g}}$ the problem formally reduces to the study of the positivity in spherical systems (see e.g. CZ18; CMP19).

\section{A1 The positivity condition for JJe models}

We now apply the previous considerations to the ellipsoidal generalization of the spherical two-component $\gamma$ models in CZ18, where

$$
\mathcal{F}(s, \theta)=\frac{1}{\xi \alpha}\left(\frac{\Omega_{\mathrm{g}}}{\Omega_{*}}\right)^{\gamma}\left(\frac{\xi+s \Omega_{\mathrm{g}}}{1+s \Omega_{*}}\right)^{4-\gamma}, \quad \alpha \equiv \frac{q_{*}}{q_{\mathrm{g}}},
$$

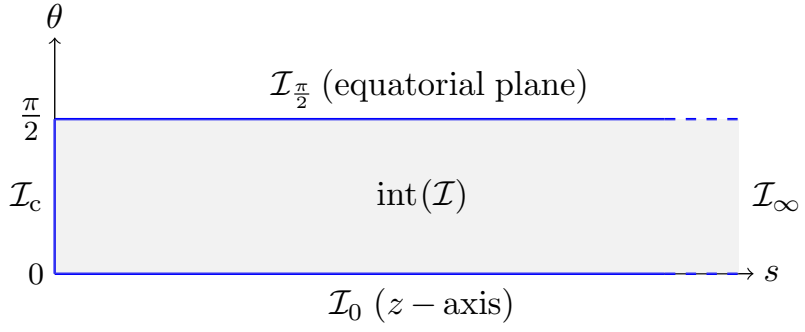

Figure A1. Illustration of the region $\mathcal{I}$ over which the function $\mathcal{F}(s, \theta)$ must be maximized in order to guarantee positivity of the DM density distribution $\rho_{\mathrm{DM}}=\mathcal{R} E_{\mathrm{g}}\left(m_{\mathrm{g}}\right)-E_{*}\left(m_{*}\right)$.

and $0 \leq \gamma<3$.

We start with the discussion of the positivity condition on the boundary of $\mathcal{I}$ (see Fig. A1). Along $\mathcal{I}_{\mathrm{c}}$,

$$
\mathcal{R}_{\mathrm{c}}=\frac{\xi^{3-\gamma}}{\alpha} \times \max _{0 \leq \theta \leq \frac{\pi}{2}} f^{\gamma}(\theta), \quad f(\theta) \equiv \frac{\Omega_{\mathrm{g}}}{\Omega_{*}},
$$

so that the problem reduces to the study of

$$
\frac{d f}{d \theta} \propto\left(1-\alpha^{2}\right) \sin 2 \theta .
$$

For $q_{*}<q_{\mathrm{g}}$ (i.e. $\alpha<1$ ), the maximum (1) is reached at $\theta=\pi / 2$, while for $q_{*}>q_{\text {g }}$ (i.e. $\alpha>1$ ) the maximum $\left(\alpha^{\gamma}\right)$ is reached at $\theta=0$. Summarizing,

$$
\mathcal{R}_{\mathrm{c}}=\xi^{3-\gamma} \times \max \left(\frac{1}{\alpha}, \alpha^{\gamma-1}\right) .
$$

Over $\mathcal{I}_{\infty}$ (i.e., for $s \rightarrow \infty$ ), from a similar analysis,

$$
\mathcal{R}_{\infty}=\frac{1}{\xi \alpha} \times \max _{0 \leq \theta \leq \frac{\pi}{2}} f^{4}(\theta)=\frac{1}{\xi} \times \max \left(\frac{1}{\alpha}, \alpha^{3}\right) .
$$

The positivity along the symmetry axis $\mathcal{I}_{0}$, and on the equatorial plane $\mathcal{I}_{\frac{\pi}{2}}$, requires

$$
\mathcal{R}_{0}=\frac{\alpha^{3}}{\xi} \sup _{s \geq 0}\left(\frac{\xi q_{\mathrm{g}}+s}{q_{*}+s}\right)^{4-\gamma}=\max \left(\frac{\alpha^{3}}{\xi}, \xi^{3-\gamma} \alpha^{\gamma-1}\right)
$$

and

$$
\mathcal{R}_{\frac{\pi}{2}}=\frac{1}{\xi \alpha} \sup _{s \geq 0}\left(\frac{\xi+s}{1+s}\right)^{4-\gamma}=\frac{1}{\alpha} \times \max \left(\frac{1}{\xi}, \xi^{3-\gamma}\right) .
$$

Finally, we consider int $(\mathcal{I})$, and, according to equation (A6), only for $q_{*}=q_{\mathrm{g}}$ (i.e., $\alpha=1$ ). Under this condition, the study of equation (A8) is trivial, and it shows that no maxima are contained in $\operatorname{int}(\mathcal{I})$, even in this case. The positivity condition in the special case $\alpha=1$ is then obtained from equation (A5), and it reads

$$
\mathcal{R} \geq \mathcal{R}_{\mathrm{m}}=\max \left(\frac{1}{\xi}, \xi^{3-\gamma}\right), \quad\left(q_{*}=q_{\mathrm{g}}\right),
$$

in agreement with the result for spherical JJ models in CZ18.

\section{A2 The positivity condition for J3e models}

Equation (A8) becomes

$$
\mathcal{F}(s, \theta)=\frac{1}{\alpha}\left(\frac{\Omega_{\mathrm{g}}}{\Omega_{*}}\right)^{2} \frac{\xi+s \Omega_{\mathrm{g}}}{\left(1+s \Omega_{*}\right)^{2}}, \quad \alpha \equiv \frac{q_{*}}{q_{\mathrm{g}}} .
$$

Repeating the same treatment of JJe models, we immediately obtain

$$
\mathcal{R}_{\mathrm{c}}=\frac{\xi}{\alpha} \times \max _{0 \leq \theta \leq \frac{\pi}{2}}\left(\frac{\Omega_{\mathrm{g}}}{\Omega_{*}}\right)^{2}=\xi \times \max \left(\frac{1}{\alpha}, \alpha\right) ;
$$


moreover, as in $\mathrm{J} 3 \mathrm{e}$ models the total density profile decreases more slowly than the stellar density for $s \rightarrow \infty$, positivity at large radii is assured independently on the value of $\mathcal{R}$, so that formally $\mathcal{R}_{\infty}=0$. Along the symmetry axis $\mathcal{I}_{0}$,

$$
\mathcal{R}_{0}=q_{\mathrm{g}} \alpha^{3} \sup _{s \geq 0} \frac{\xi q_{\mathrm{g}}+s}{\left(q_{*}+s\right)^{2}}=\alpha \times \begin{cases}\frac{\alpha^{2}}{4(\alpha-\xi)}, & \xi \leq \frac{\alpha}{2}, \\ \xi, & \xi \geq \frac{\alpha}{2},\end{cases}
$$

and, along the equatorial plane $\mathcal{I}_{\frac{\pi}{2}}$,

$$
\mathcal{R}_{\frac{\pi}{2}}=\frac{1}{\alpha} \sup _{s \geq 0} \frac{\xi+s}{(1+s)^{2}}=\frac{1}{\alpha} \times \begin{cases}\frac{1}{4(1-\xi)}, & \xi \leq \frac{1}{2}, \\ \xi, & \xi \geq \frac{1}{2} .\end{cases}
$$

For what concerns the positivity in the interior of $\mathcal{I}$, the only case to be considered is $q_{*}=q_{\mathrm{g}}$ (i.e., $\alpha=1$ ). It is easy to show that $\mathcal{F}$ has no critical points in $\operatorname{int}(\mathcal{I})$ when $\xi>1 / 2$; for $\xi \leq 1 / 2$, instead, $\mathcal{R}_{\text {int }}=1 /[4(1-\xi)]$. In conclusion, for $\alpha \neq 1, \mathcal{R}$ is obtained from equation (A5) and the previous results, while in the special case $\alpha=1$ the final condition is

$$
\mathcal{R} \geq \mathcal{R}_{\mathrm{m}}=\left\{\begin{array}{ll}
\frac{1}{4(1-\xi)}, & \xi \leq \frac{1}{2}, \\
\xi, & \xi \geq \frac{1}{2},
\end{array} \quad\left(q_{*}=q_{\mathrm{g}}\right),\right.
$$

in agreement with the result for spherical J3 models in CMP19.

\section{APPENDIX B: HOMOEOIDAL EXPANSION}

A thorough description of the homoeoidal expansion method can be found in CB05. Here we just report the formulae strictly needed for the present work. Consider an ellipsoidal mass density distribution $\rho$ stratified, in Cartesian coordinates, over surfaces labelled by

$$
m^{2} \equiv \frac{x^{2}}{a^{2}}+\frac{y^{2}}{b^{2}}+\frac{z^{2}}{c^{2}}=\frac{x^{2}}{a^{2}}+\frac{y^{2}}{a^{2}(1-\epsilon)^{2}}+\frac{z^{2}}{a^{2}(1-\eta)^{2}}
$$

where $a \geq b \geq c>0, b / a \equiv 1-\epsilon$, and $c / a \equiv 1-\eta$; when $\epsilon=\eta=0, m=s=r / a$. We write

$$
\rho(m)=\rho_{\mathrm{n}} \times \frac{\tilde{\rho}(m)}{(1-\epsilon)(1-\eta)}, \quad \rho_{\mathrm{n}} \equiv \frac{M_{\mathrm{n}}}{4 \pi a^{3}},
$$

where $\rho_{\mathrm{n}}$ is a normalization density, and $M_{\mathrm{n}}$ is the mass of the ellipsoid contained inside the arbitrary ellipsoid defined by $m$. With this choice, $M_{\mathrm{n}}$ is independent of the adopted flattenings, the socalled constrained case. Of course, for a model of finite total mass, the natural choice is to adopt for $M_{\mathrm{n}}$ the total mass. Note that, for a model of finite total mass $M$, the normalization assures that the total mass is conserved independently of the value of $\epsilon$ and $\eta$. In case of an infinite total mass (such as $\rho_{\mathrm{g}}$ in J3e models), the condition assures that the mass contained inside any $m$ is conserved. The oblate axisymmetric models discussed in this paper (see Section 2.2) are obtained for $\epsilon=0, a=r_{*}, \eta=1-q_{*}$ for the stellar component, and $\eta=1-q_{\mathrm{g}}$ for the total density.

By expanding at the linear order in terms of the flattenings one obtains

$$
\frac{\tilde{\rho}(m)}{(1-\epsilon)(1-\eta)}=\tilde{\varrho}_{0}(s)+(\epsilon+\eta) \tilde{\varrho}_{1}(s)+\left(\epsilon \tilde{y}^{2}+\eta \tilde{z}^{2}\right) \tilde{\varrho}_{2}(s)
$$

where $\tilde{y} \equiv y / a, \tilde{z} \equiv z / a$, and

$$
\tilde{\varrho}_{0}(s)=\tilde{\varrho}_{1}(s)=\tilde{\rho}(s), \quad \tilde{\varrho}_{2}(s)=\frac{1}{s} \frac{d \tilde{\rho}(s)}{d s} .
$$

In order to be physically acceptable, the expanded density must be nowhere negative, and this requirement sets an upper limit on the possible values of $\epsilon$ and $\eta$, as a function of the specific density profile adopted. By changing variables to spherical coordinates, and following the approach introduced in Appendix A, it can be shown that positivity of equation (B3) for $0 \leq \epsilon \leq \eta<1$, and for a monothonically decreasing $\tilde{\rho}(s)$, is assured provided that

$$
\epsilon \geq\left(A_{M}-1\right) \eta-1, \quad A_{M} \equiv \sup _{s \geq 0}\left|\frac{d \ln \tilde{\rho}(s)}{d \ln s}\right| .
$$

For $\gamma$ models, $A_{M}=4$, so that in the axysimmetric case $(\epsilon=0)$ we recover the condition $\eta \leq 1 / 3$ (see CB05).

The general quadrature formula for the potential of a density distribution $\rho(m)$ is given by

$$
\Psi(\mathbf{x})=\pi a b c G \int_{0}^{\infty} \frac{\Delta \Psi[m(\mathbf{x} ; \tau)]}{\sqrt{\left(a^{2}+\tau\right)\left(b^{2}+\tau\right)\left(c^{2}+\tau\right)}} d \tau,
$$

(see e.g. Kellogg 1953; Chandrasekhar 1969; BT08), where

$$
\Delta \Psi[m(\mathbf{x} ; \tau)] \equiv 2 \int_{m(\mathbf{x} ; \tau)}^{\infty} \rho(m) m d m
$$

and

$$
m^{2}(\mathbf{x} ; \tau) \equiv \frac{x^{2}}{a^{2}+\tau}+\frac{y^{2}}{b^{2}+\tau}+\frac{z^{2}}{c^{2}+\tau}
$$

note that the variable $\tau$ has the dimension of a squared length.

By inserting equation (B2) in equation (B6), and after normalization of all lengths to $a$ (and $\tau$ to $a^{2}$ ), it is immediate to show that

$$
\Psi(\mathbf{x})=\Psi_{\mathrm{n}} \times \tilde{\Psi}(\mathbf{x}), \quad \Psi_{\mathrm{n}} \equiv \frac{G M_{\mathrm{n}}}{a},
$$

where the meaning of the function $\tilde{\Psi}$ is obvious. Expanding the integrand in equation (B6) at linear order in the flattenings, and inverting order of integration, some algebra shows that

$$
\tilde{\Psi}(\mathbf{x})=\tilde{\psi}_{0}(s)+(\epsilon+\eta) \tilde{\psi}_{1}(s)+\left(\epsilon \tilde{y}^{2}+\eta \tilde{z}^{2}\right) \tilde{\psi}_{2}(s),
$$

where

$$
\tilde{\psi}_{i}(s)=\left\{\begin{array}{l}
\frac{1}{s} \int_{0}^{s} \tilde{\rho}(m) m^{2} d m+\int_{s}^{\infty} \tilde{\rho}(m) m d m \\
\frac{1}{3 s^{3}} \int_{0}^{s} \tilde{\rho}(m) m^{4} d m+\frac{1}{3} \int_{s}^{\infty} \tilde{\rho}(m) m d m \\
-\frac{1}{s^{5}} \int_{0}^{s} \tilde{\rho}(m) m^{4} d m
\end{array}\right.
$$

with $i=0,1,2$, respectively. Then, Poisson's equation for the dimensionless potential-density pair $(\tilde{\Psi}, \tilde{\rho})$ becomes

$$
\tilde{\nabla}^{2} \tilde{\Psi}(\mathbf{x})=-\frac{\tilde{\rho}(m)}{(1-\epsilon)(1-\eta)}, \quad \tilde{\nabla}^{2} \equiv a^{2} \nabla^{2} .
$$

The previous formulae, in the axisymmetric oblate case, are obtaines by setting $\epsilon=0$ and $0<\eta<1$. It may be convenient in some computation to recast equations (B3) and (B10) in terms of $R^{2}$ instead of $z^{2}=r^{2}-R^{2}$, and in this case the corresponding functions are given by

$$
\tilde{\rho}_{0}=\tilde{\varrho}_{0}, \quad \tilde{\rho}_{1}=\tilde{\varrho}_{1}+s^{2} \tilde{\varrho}_{2}, \quad \tilde{\rho}_{2}=-\tilde{\varrho}_{2},
$$

and

$$
\tilde{\Psi}_{0}=\tilde{\psi}_{0}, \quad \tilde{\Psi}_{1}=\tilde{\psi}_{1}+s^{2} \tilde{\psi}_{2}, \quad \tilde{\Psi}_{2}=-\tilde{\psi}_{2} .
$$

For example, when computing properties on the equatorial plane, where $z=0$, such as for example in the derivation of the circular speed $v_{\mathrm{c}}(R)$, angular momentum $J_{\mathrm{c}}(R)$, or radial epicyclic 
frequency $\kappa_{R}(R)$, it is useful to work with the "explicit- $z$ formulation", while in some other case, such as the integration of the Jeans equations, or the derivation of the vertical epicyclic frequency $\kappa_{z}(R)$, it is more useful to use the "explicit- $R$ formulation". In particular, we recall that $\kappa_{R}$ and $\kappa_{z}$ are defined as

$$
\kappa_{R}^{2}(R) \equiv \frac{1}{R^{3}} \frac{d J_{\mathrm{c}}^{2}}{d R}, \quad \kappa_{z}^{2}(R) \equiv-\left(\frac{\partial^{2} \Psi}{\partial z^{2}}\right)_{z=0} .
$$

By defining $\kappa_{\mathrm{n}}^{2} \equiv \Psi_{\mathrm{n}} / a^{2}$, simple algebra shows that

$$
\frac{\kappa_{z}^{2}(R)}{\kappa_{\mathrm{n}}^{2}}=\tilde{\kappa}_{z 0}^{2}(\tilde{R})+\eta \tilde{\kappa}_{z 1}^{2}(\tilde{R})+\eta \tilde{R}^{2} \tilde{\kappa}_{z 2}^{2}(\tilde{R}),
$$

where

$$
\tilde{\kappa}_{z i}^{2}(\tilde{R}) \equiv-\frac{1}{\tilde{R}} \frac{d \tilde{\Psi}_{i}(\tilde{R})}{d \tilde{R}}, \quad(i=0,1,2),
$$

and

$$
\frac{\kappa_{R}^{2}(R)}{\kappa_{\mathrm{n}}^{2}}=\tilde{\kappa}_{R 0}^{2}(\tilde{R})+\eta \tilde{\kappa}_{R 1}^{2}(\tilde{R})
$$

with

$$
\tilde{\kappa}_{R i}^{2}(\tilde{R}) \equiv-\frac{1}{\tilde{R}^{3}} \frac{d}{d \tilde{R}}\left[\tilde{R}^{3} \frac{d \tilde{\psi}_{i}(\tilde{R})}{d \tilde{R}}\right], \quad(i=0,1) .
$$




\section{APPENDIX C: VELOCITY DISPERSION}

We report here the explicit expressions of the functions entering the velocity dispersion profiles in Section 4.

\section{C1 JJe models}

The three functions describing the $\mathrm{BH}$ contribution in equation (38) are

$$
\begin{gathered}
A(s) \equiv \int_{s}^{\infty} \frac{\tilde{\rho}_{* 0}}{s^{\prime 2}} d s^{\prime}=\frac{12 s^{3}+6 s^{2}-2 s+1}{3 s^{3}(1+s)}+4 \ln \frac{s}{1+s} \\
B(s) \equiv \int_{s}^{\infty} \frac{\tilde{\rho}_{* 1}}{s^{\prime 2}} d s^{\prime}=\frac{24 s^{4}+36 s^{3}+8 s^{2}-2 s-1}{3 s^{3}(1+s)^{2}}+8 \ln \frac{s}{1+s}, \\
C(s) \equiv \int_{s}^{\infty} \frac{\tilde{\rho}_{* 2}}{s^{\prime 2}} d s^{\prime}=-\frac{180 s^{6}+270 s^{5}+60 s^{4}-15 s^{3}+6 s^{2}-3 s-4}{10 s^{5}(1+s)^{2}}-18 \ln \frac{s}{1+s} .
\end{gathered}
$$

Note that from equation (16) it follows that $A>0, B<0$ and $C>0$.

For the contribution of the galaxy to the velocity dispersion in equation (39), an elementary integration leads to

$$
\begin{aligned}
& D(s) \equiv-\int_{s}^{\infty} \tilde{\rho}_{* 0} \frac{d \tilde{\Psi}_{\mathrm{g} 0}}{d s^{\prime}} d s^{\prime}=-\frac{3 \xi^{2}-\xi-1}{\xi^{2}(\xi-1)(1+s)}-\frac{(3 \xi+2) s-\xi}{2 \xi^{2} s^{2}(1+s)}-\frac{1}{\xi^{3}(\xi-1)^{2}} \ln \frac{s}{\xi+s}-\frac{3 \xi-4}{(\xi-1)^{2}} \ln \frac{s}{1+s}, \\
& E(s) \equiv-\int_{s}^{\infty} \tilde{\rho}_{* 1} \frac{d \tilde{\Psi}_{\mathrm{g} 0}}{d s^{\prime}} d s^{\prime}=-\frac{2\left(3 \xi^{3}-6 \xi^{2}+2 \xi-1\right) s+9 \xi^{3}-18 \xi^{2}+9 \xi-4}{2 \xi^{2}(\xi-1)^{2}(1+s)^{2}}-\frac{2(\xi-1) s+\xi}{2 \xi^{2} s^{2}(1+s)^{2}} \\
& +\frac{3 \xi-1}{\xi^{3}(\xi-1)^{3}} \ln \frac{s}{\xi+s}-\frac{3 \xi^{2}-9 \xi+8}{(\xi-1)^{3}} \ln \frac{s}{1+s}, \\
& F(s) \equiv-\int_{s}^{\infty} \tilde{\rho}_{* 2} \frac{d \tilde{\Psi}_{\mathrm{g} 0}}{d s^{\prime}} d s^{\prime}=\frac{2\left(5 \xi^{5}-8 \xi^{4}+\xi^{3}+\xi^{2}+\xi-1\right) s+15 \xi^{5}-24 \xi^{4}+3 \xi^{3}+3 \xi^{2}+5 \xi-4}{\xi^{4}(\xi-1)^{2}(1+s)^{2}} \\
& +\frac{4\left(5 \xi^{3}+2 \xi^{2}-3\right) s^{3}-\xi\left(5 \xi^{2}+2 \xi-6\right) s^{2}+2 \xi^{2}(\xi-2) s+3 \xi^{3}}{6 \xi^{4} s^{4}(1+s)^{2}}-\frac{2(2 \xi-1)}{\xi^{5}(\xi-1)^{3}} \ln \frac{s}{\xi+s}+\frac{2\left(5 \xi^{2}-13 \xi+9\right)}{(\xi-1)^{3}} \ln \frac{s}{1+s}, \\
& G(s) \equiv-\int_{s}^{\infty} \tilde{\rho}_{* 0} \frac{d \tilde{\Psi}_{\mathrm{g} 1}}{d s^{\prime}} d s^{\prime}=\left[24 \ln \frac{s}{1+s}+\frac{120 s^{5}+60 s^{4}-20 s^{3}+10 s^{2}-6 s+4}{5 s^{5}(1+s)}\right] \xi^{2} \ln \frac{\xi+s}{\xi}+\frac{5 \xi^{2}+10 \xi+9}{5 \xi^{3}} \ln \frac{\xi+s}{s} \\
& +\frac{\xi\left(24 \xi^{3}-60 \xi^{2}+43 \xi-5\right)}{(\xi-1)^{3}} \ln \frac{\xi+s}{1+s}-\frac{1}{\xi^{2}(\xi-1)^{2}(\xi+s)}+\frac{\xi\left(4 \xi^{2}-6 \xi+1\right)}{(\xi-1)^{2}(1+s)} \\
& +\frac{4\left(20 \xi^{3}+5 \xi^{2}-2\right) s^{3}-\xi\left(40 \xi^{2}+10 \xi+1\right) s^{2}+4 \xi^{2}(5 \xi+1) s-8 \xi^{3}}{10 \xi^{2} s^{4}}-24 \xi^{2} \mathcal{H}(\xi, s), \\
& H(s) \equiv-\int_{s}^{\infty} \tilde{\rho}_{* 0} \frac{d \tilde{\Psi}_{\mathrm{g} 2}}{d s^{\prime}} d s^{\prime}=\left[80 \ln \frac{1+s}{s}-\frac{1680 s^{7}+840 s^{6}-280 s^{5}+140 s^{4}-84 s^{3}+56 s^{2}-40 s+30}{21 s^{7}(1+s)}\right] \xi^{2} \ln \frac{\xi+s}{\xi} \\
& +\frac{2\left(35 \xi^{4}+21 \xi^{3}-14 \xi-15\right)}{21 \xi^{5}} \ln \frac{\xi+s}{s}-\frac{2 \xi\left(40 \xi^{3}-100 \xi^{2}+75 \xi-14\right)}{(\xi-1)^{3}} \ln \frac{\xi+s}{1+s}+\frac{1}{\xi^{4}(\xi-1)^{2}(\xi+s)} \\
& -\frac{\xi\left(10 \xi^{2}-15 \xi+4\right)}{(\xi-1)^{2}(1+s)}-\frac{630 \xi^{5}+175 \xi^{4}+126 \xi^{3}+63 \xi^{2}+14 \xi-9}{21 \xi^{4} s}+80 \xi^{2} \mathcal{H}(\xi, s) \\
& +\frac{6\left(350 \xi^{4}+105 \xi^{3}+63 \xi^{2}+28 \xi+6\right) s^{4}-2 \xi\left(630 \xi^{3}+189 \xi^{2}+98 \xi+33\right) s^{3}+3 \xi^{2}\left(252 \xi^{2}+70 \xi+27\right) s^{2}-30 \xi^{3}(14 \xi+3) s+180 \xi^{4}}{126 \xi^{3} s^{6}} .
\end{aligned}
$$


C1.1 The case $\xi=1$

$$
\begin{gathered}
D(s)=-\frac{\left(6 s^{2}+6 s-1\right)(2 s+1)}{2 s^{2}(1+s)^{2}}-6 \ln \frac{s}{1+s}, \\
E(s)=-\frac{12 s^{4}+30 s^{3}+22 s^{2}+3 s+3}{6 s^{2}(1+s)^{3}}-2 \ln \frac{s}{1+s}, \\
F(s)=\frac{60 s^{6}+150 s^{5}+110 s^{4}+15 s^{3}-3 s^{2}+s+3}{6 s^{4}(1+s)^{3}}+10 \ln \frac{s}{1+s}, \\
G(s)=\left[24 \ln \frac{s}{1+s}+\frac{120 s^{5}+60 s^{4}-20 s^{3}+10 s^{2}-6 s+4}{5 s^{5}(1+s)}\right] \ln (1+s)-\frac{24}{5} \ln \frac{s}{1+s} \\
+\frac{576 s^{6}+1260 s^{5}+716 s^{4}+9 s^{3}-9 s^{2}-24}{30 s^{4}(1+s)^{3}}-24 \mathcal{H}(1, s), \\
-\frac{10404 s^{8}+23490 s^{7}+14314 s^{6}+711 s^{5}-243 s^{4}+109 s^{3}-57 s^{2}-30 s-180}{126 s^{6}(1+s)^{3}}+80 \mathcal{H}(1, s) .
\end{gathered}
$$

\section{C2 J3e models}

The functions $A, B$ and $C$ are the same as for J3e models. The functions from $D$ to $H$ are instead given by

$$
\begin{aligned}
& D(s)=A(s) \ln \frac{\xi+s}{\xi}+\frac{9 \xi^{2}+3 \xi+1}{3 \xi^{3}} \ln \frac{\xi+s}{s}+\frac{1}{\xi-1} \ln \frac{\xi+s}{1+s}-\frac{2(1+3 \xi) s-\xi}{6 \xi^{2} s^{2}}-4 \mathcal{H}(\xi, s), \\
& E(s)=B(s) \ln \frac{\xi+s}{\xi}+\frac{9 \xi^{2}-1}{3 \xi^{3}} \ln \frac{\xi+s}{s}+\frac{5 \xi-6}{(\xi-1)^{2}} \ln \frac{\xi+s}{1+s}+\frac{3 \xi^{2}+\xi-1}{3 \xi^{2}(\xi-1)(1+s)}-\frac{(\xi-2) s+\xi}{6 \xi^{2} s^{2}(1+s)}-8 \mathcal{H}(\xi, s), \\
& F(s)=C(s) \ln \frac{\xi+s}{\xi}-\frac{100 \xi^{4}+20 \xi^{3}-5 \xi-4}{10 \xi^{5}} \ln \frac{\xi+s}{s}-\frac{8 \xi-9}{(\xi-1)^{2}} \ln \frac{\xi+s}{1+s}+\frac{10 \xi^{4}-20 \xi^{3}-5 \xi^{2}+\xi+4}{10 \xi^{4}(\xi-1)(1+s)} \\
& +\frac{3\left(40 \xi^{3}+5 \xi^{2}-6 \xi-8\right) s^{3}+\xi(5 \xi+4)(3-2 \xi) s^{2}-4 \xi^{2}(\xi+2) s+6 \xi^{3}}{60 \xi^{4} s^{4}(1+s)}+18 \mathcal{H}(\xi, s) \\
& G(s)=\left[12 \ln \frac{1+s}{s}-\frac{60 s^{5}+30 s^{4}-10 s^{3}+5 s^{2}-3 s+2}{5 s^{5}(1+s)}\right] \xi^{2} \ln \frac{\xi+s}{\xi}+\frac{5 \xi^{2}+5 \xi+3}{5 \xi^{3}} \ln \frac{\xi+s}{s}-\frac{\xi\left(12 \xi^{2}-18 \xi+5\right)}{(\xi-1)^{2}} \ln \frac{\xi+s}{1+s} \\
& -\frac{30 \xi^{4}-20 \xi^{3}-2 \xi-3}{5 \xi^{2}(\xi-1)(1+s)}-\frac{\left(20 \xi^{3}+5 \xi^{2}+7 \xi+6\right) s^{3}-\xi\left(10 \xi^{2}+3 \xi+3\right) s^{2}+2 \xi^{2}(3 \xi+1) s-4 \xi^{3}}{10 \xi^{2} s^{4}(1+s)}+12 \xi^{2} \mathcal{H}(\xi, s), \\
& H(s)=\left[40 \ln \frac{s}{1+s}+\frac{840 s^{7}+420 s^{6}-140 s^{5}+70 s^{4}-42 s^{3}+28 s^{2}-20 s+15}{21 s^{7}(1+s)}\right] \xi^{2} \ln \frac{\xi+s}{\xi}+\frac{70 \xi^{4}+21 \xi^{3}-7 \xi-6}{21 \xi^{5}} \ln \frac{\xi+s}{s} \\
& +\frac{40 \xi^{3}-60 \xi^{2}+10 \xi+9}{(\xi-1)^{2}} \ln \frac{\xi+s}{1+s}+\frac{420 \xi^{6}-280 \xi^{5}-140 \xi^{4}+21 \xi^{3}+7 \xi^{2}-\xi-6}{21 \xi^{4}(\xi-1)(1+s)} \\
& +\frac{280 \xi^{5}+70 \xi^{4}-42 \xi^{3}-7 \xi^{2}+8 \xi+12}{42 \xi^{4} s(1+s)}-40 \xi^{2} \mathcal{H}(\xi, s) \\
& -\frac{\left(420 \xi^{4}+126 \xi^{3}-14 \xi^{2}+9 \xi+18\right) s^{4}-\xi\left(252 \xi^{3}+84 \xi^{2}+5 \xi+12\right) s^{3}+3 \xi^{2}\left(56 \xi^{2}+20 \xi+3\right) s^{2}-15 \xi^{3}(8 \xi+3) s+90 \xi^{4}}{126 \xi^{3} s^{6}(1+s)} .
\end{aligned}
$$


C2.1 The case $\xi=1$

$$
\begin{gathered}
D(s)=A(s) \ln (1+s)+\frac{13}{3} \ln \frac{1+s}{s}-\frac{2 s^{2}+7 s-1}{6 s^{2}(1+s)}-4 \mathcal{H}(1, s), \\
E(s)=B(s) \ln (1+s)+\frac{8}{3} \ln \frac{1+s}{s}+\frac{32 s^{3}+36 s^{2}-1}{6 s^{2}(1+s)^{2}}-8 \mathcal{H}(1, s), \\
F(s)=C(s) \ln (1+s)-\frac{111}{10} \ln \frac{1+s}{s}-\frac{138 s^{5}+117 s^{4}-34 s^{3}+s^{2}+2 s-2}{20 s^{4}(1+s)^{2}}+18 \mathcal{H}(1, s), \\
G(s)=\left[12 \ln \frac{1+s}{s}-\frac{60 s^{5}+30 s^{4}-10 s^{3}+5 s^{2}-3 s+2}{5 s^{5}(1+s)}\right] \ln (1+s)+\frac{13}{5} \ln \frac{1+s}{s} \\
H(s)=\left[\begin{array}{c}
146 s^{5}+189 s^{4}+22 s^{3}-8 s^{2}+4 s-4 \\
10 s^{4}(1+s)^{2}
\end{array}\right. \\
\left.\frac{s}{1+s}+\frac{840 s^{7}+420 s^{6}-140 s^{5}+70 s^{4}-42 s^{3}+28 s^{2}-20 s+15}{21 s^{7}(1+s)}\right] \ln (1+s)+\frac{26}{7} \ln \frac{1+s}{s} \\
+\frac{4572 s^{7}+5598 s^{6}+404 s^{5}-206 s^{4}+116 s^{3}-72 s^{2}+75 s-90}{126 s^{6}(1+s)^{2}}-40 \mathcal{H}(1, s) .
\end{gathered}
$$

\section{C3 Functions needed for the projected velocity dispersion}

The asymptotic behaviour of $\sigma_{\text {los }}^{2}$ in equations (61) and (63) near the center, at the linear order in the flattenings, is determined by the functions

$$
\begin{gathered}
a_{\mathrm{g}}(\tilde{X}, \tilde{Y})=\pi \frac{6 \tilde{R}^{2}+3 \eta_{*}\left(2 \tilde{Y}^{2}-\tilde{X}^{2}\right)+\eta_{\mathrm{g}}\left(3 \tilde{X}^{2}+2 \tilde{Y}^{2}\right)}{12 \xi \tilde{R}^{2}}-k^{2} \frac{4\left(3 \eta_{*}-\eta_{\mathrm{g}}\right) \tilde{Y}^{2}}{3 \xi \pi \tilde{R}^{2}} \\
a_{\mathrm{BH}}(\tilde{X}, \tilde{Y})=\frac{10 \tilde{R}^{2}+6 \eta_{*}\left(3 \tilde{Y}^{2}-\tilde{X}^{2}\right)}{15 \tilde{R}^{2}}-k^{2} B^{2}\left(\frac{1}{2}, \frac{5}{4}\right) \frac{4 \eta_{*} \tilde{Y}^{2}}{5 \pi \tilde{R}^{2}}
\end{gathered}
$$

while at large radii

$$
b(\tilde{X}, \tilde{Y})=\frac{7 \tilde{R}^{2}+\eta_{*}\left(31 \tilde{Y}^{2}-17 \tilde{X}^{2}\right)}{15 \tilde{R}^{2}}-k^{2} B^{2}\left(\frac{1}{2}, \frac{9}{4}\right) \frac{4 \eta_{*} \tilde{Y}^{2}}{\pi \tilde{R}^{2}}
$$

